hep-th/0212061

MCTP-02-45

$\mathrm{UW} / \mathrm{PT} 02-28$

CERN-TH/02-211

\title{
A Soluble String Theory of Hadrons
}

\author{
E.G. Gimon ${ }^{1}$, L.A. Pando Zayas ${ }^{1,2}$, J. Sonnenschein ${ }^{1,3,4}$ and M.J. Strassler ${ }^{1,5}$ \\ ${ }^{1}$ School of Natural Sciences, Institute for Advanced Study \\ Princeton, NJ 08540 \\ ${ }^{2}$ Michigan Center for Theoretical Physics \\ Randall Laboratory of Physics, The University of Michigan \\ Ann Arbor, MI 48109-1120 \\ ${ }^{3}$ School of Physics and Astronomy \\ Beverly and Raymond Sackler Faculty of Exact Sciences \\ Tel Aviv University, Ramat Aviv, 69978, Israel \\ ${ }^{4}$ Theory Division, CERN \\ CH-1211 Geneva 23, Switzerland \\ ${ }^{5}$ Department of Physics and Astronomy \\ P.O Box 351560, University of Washington \\ Seattle, WA 98195
}

\begin{abstract}
We consider Penrose limits of the Klebanov-Strassler and Maldacena-Núñez holographic duals to $\mathcal{N}=1$ supersymmetric Yang-Mills. By focusing in on the IR region we obtain exactly solvable string theory models. These represent the nonrelativistic motion and low-lying excitations of heavy hadrons with mass proportional to a large global charge. We argue that these hadrons, both physically and mathematically, take the form of heavy nonrelativistic strings; we term them "annulons." A simple toy model of a string boosted along a compact circle allows us considerable insight into their properties. We also calculate the Wilson loop carrying large global charge and show the effect of confinement is quadratic, not linear, in the string tension.
\end{abstract}




\section{Introduction and Summary}

Since the experimental observation of stringy behavior in hadronic physics, it has been hoped that these aspects of the strong interactions could be predicted from an as-yet-unknown string theory. The recent discovery of a precise duality of gauge theory and string theory has allowed some progress. The string theoretic descriptions of several confining gauge theories with a large number of colors have now been found. More precisely, the backgrounds on which the dual type IIB strings propagate are now known. However, the string theory on these backgrounds is not soluble. This is unfortunate: while the masses of low-lying low-spin hadrons can be computed from supergravity (SUGRA), the full hadron spectrum requires string theory. SUGRA cannot describe states of high spin and cannot see the Regge trajectories that we would expect gauge theory to exhibit.

Initially most information gained from gauge/string duality was obtained through analysis of the SUGRA backgrounds, or of semiclassical branes and/or strings in these backgrounds. However, this has begun to change [1-3]. The remarkable work of Berenstein, Maldacena and Nastase (BMN) [2] has provided a link between an exactly solvable worldsheet string theory [4] and a sector of the conformal $\mathcal{N}=4$ supersymmetric Yang-Mills (SYM) theory. This work was extended to other conformal theories in [5-7]. Attempts to apply the Penrose limit [8-10] to non-conformal backgrounds $[7,11,12]$ have resulted in string theories with world-sheet timedependent mass terms. The geodesics chosen in these cases are appropriate to the study of the properties of the renormalization-group $(\mathrm{RG})$ flow. The corresponding world-line problem in the Pilch-Warner background solution is exactly solvable [11], giving the "branching" of a given operator in the ultraviolet $(\mathrm{UV}) \mathcal{N}=4 \mathrm{SYM}$ into operators of the infrared (IR) $\mathcal{N}=1$ theory (the conformal theory with two adjoint chiral multiplets.)

In this paper we study theories that exhibit confinement and a discrete spectrum of hadrons in the IR. Our interest is not in operators but in hadronic states of fixed mass in Minkowski space; consequently we choose a different type of geodesic as a basis for our Penrose limit. By focusing on geodesics that are frozen at the minimal $A d S$ radius in the IR, and that spiral inside the cylinder formed by the Minkowski time direction and a circle in the compact part of the ten dimensional space, we can obtain an exactly solvable time-independent string theory background in the Penrose limit which captures the dynamics of hadrons with a large global charge.

The specific confining gauge theories we consider consist of $\mathcal{N}=1 \mathrm{SYM}$ plus massive particles in the adjoint representation and carrying a global abelian charge. The string Hamiltonian describes hadrons which are bound states of these massive particles, in the limit that the global charge and the number of colors both go to infinity. Roughly speaking, the string sigma model takes the form of a ten-dimensional string, which in light-cone gauge is "compactified" by world-sheet mass terms down to the three massless spatial dimensions of Minkowski space. The vacuum of the string theory is a stationary hadron of large mass and charge. Our string Hamiltonian describes its non-relativistic motion (and that of its fermionic superpartners) in three spatial dimensions, and its low-lying stringy excitations in those directions, as well as excitations which add a small number of other globally-charged constituents. We argue that these hadrons take the physical form of non-relativistic strings. 
In backgrounds corresponding to confining gauge theories, there is a minimum $A d S$ radius $r_{0}$, where $g_{t t}$ generally goes to a non-zero minimum. In section 2 we use this, along with mild constraints on the space perpendicular to the branes, to find null geodesics fixed at $r_{0}$ (other "frozen" geodesics appear in [17]). We explicitly discuss the two trademark SUGRA solutions dual to $\mathcal{N}=1 \mathrm{SYM}$ in the IR: the Maldacena-Núñez (MN) background [13], in Section 3, and the Klebanov-Strassler (KS) [14,15] background (with a nonstandard but convenient parameterization), in Section 4. Section 5 describes the light-cone quantization and spectrum of the corresponding string theories, with comments about the unbroken supersymmetries. In Section 6 we find a hadronic interpretation of the string spectrum for the KS case. (The MN case is similar but less well understood.) In particular, we show that a very simple toy model (a string moving on a compact circle) captures some of the terms of the string Hamiltonian, thereby emphasizing its universality.

In section 7 we obtain, under very general assumptions, an expression for the Wilson loop with global charge. We arrive at the same formula for the Wilson loop using heuristic field theory arguments, the above-mentioned toy model, and a semi-classical string analysis. We close with a few comments and include three appendices. Appendix A contains an explicit derivation of the new parameterization of the deformed conifold and its relation to the standard coordinates. In appendix B we present the main steps in the derivation of the string Hamiltonian. Appendix C contains some technical arguments about the reliability of the Wilson loop ansatz used in section 6 .

\section{Null geodesics in the IR of confining theories}

Let us first clarify why we choose to study null geodesics at the minimal $A d S$ radius. The essential feature of a confining theory is that it has stable electric flux tubes and a spectrum of hadrons of definite (four-dimensional) mass. A hadron of definite four-dimensional mass is a supergravity eigenstate of the ten-dimensional Laplacian which is also an eigenstate of the fourdimensional Minkowski Laplacian. (This is in contrast to an operator of definite dimension, which is an eigenstate of the five-dimensional $A d S$ Laplacian.) These states are plane waves in the Minkowski directions and have nontrivial wave functions $\psi(r, \Omega)$ on the remaining six directions; here $r$ is the $A d S$ radius (which extends from the boundary at $r \rightarrow \infty$ to a finite minimum at $r=r_{0}>0$.) A hadron's wave function falls off as $r^{-\Delta}$, where $\Delta$ is the dimension of the lowest-dimension operator which can create the hadron. A hadron of large charge $J$ - which is typically heavy, $m \sim J$, since it has many constituents of charge 1 - can be created only by an operator of large charge, which, since it contains of order $J$ fields, has $\Delta \sim J$. Consequently hadrons of high charge correspond to modes which are concentrated close to $r=r_{0}{ }^{1}$

Since these phenomena are localized at $r=r_{0}$, we should expect they are sensitive mainly to the IR physics of the gauge theory, and should not depend much on the UV completion of the low-energy theory. For this reason, we expect only mild differences between the MN and

${ }^{1}$ This is one of many examples which caution that one must avoid naive application of the dictum that $A d S$ radius is the same as energy; baryons represent another. 
KS examples, which are both $\mathcal{N}=1 \mathrm{SYM}$ in the IR; although their geometries differ greatly in the UV, they are similar in the IR and we would expect the plane wave string theories are also similar. In either background, it is natural to look for geodesics with $r=r_{0}$ and $\dot{r}=0, \dot{t}=1$, where a dot represents a derivative with respect to the affine parameter of the geodesic. With this choice, the pp-wave Hamiltonian will measure not dimensions of operators (alternatively, energies of states on a spatial $S^{3}$ ) but rather energies of states in Minkowski space (with a flat spatial $\mathbf{R}^{3}$.)

In order for a geodesic at a fixed radius to be null (the key ingredient for a consistent Penrose limit), it must move both in time and in a bulk spatial direction. Requiring isotropy in the three spatial dimensions of the gauge theory, along with $\dot{r}=0$, forces us to choose the geodesic to move on a curve inside the other bulk dimensions, typically a closed circle generated by a Killing vector. Consequently the states in the dual gauge theory will carry large charge under the corresponding global symmetry. Their spins, by contrast, will be of order one.

The conditions for a null geodesic of this type are easily found. The time $t$ and the radial direction $r$ are effectively described by the following Lagrangian

$$
\mathcal{L}=-g_{t t} \dot{t}^{2}+g_{r r} \dot{r}^{2}+g_{\phi \phi} \dot{\phi}^{2}
$$

where dot means differentiation with respect to the affine parameter $u$. Assuming for simplicity that the metric depends only on the radial coordinate (which is approximately true in some neighborhood of interest), the equations of motions are

$$
\begin{aligned}
\dot{t} & =\frac{E}{g_{t t}}, \quad \dot{\phi}=\frac{\mu}{g_{\phi \phi}}, \\
2 \frac{d}{d u}\left(g_{r r} \dot{r}\right) & =\dot{r}^{2} \partial_{r} g_{r r}-\dot{t}^{2} \partial_{r} g_{t t}+\dot{\phi}^{2} \partial_{r} g_{\phi \phi} .
\end{aligned}
$$

There is also a constraint, $\mathcal{L}=0$, which we re-write using the equations of motion for $t$ and $\phi$

$$
g_{r r} \dot{r}^{2}=\frac{E^{2}}{g_{t t}}-\frac{\mu^{2}}{g_{\phi \phi}} .
$$

Since we are interested in geodesics at a fixed radius $r=r_{0}$ we impose the condition $\left.\dot{r}\right|_{r_{0}}=0$. Thus (2.3) tells us that in the neighborhood of $r_{0}$ we have

$$
\frac{E^{2}}{g_{t t}}=\frac{\mu^{2}}{g_{\phi \phi}} .
$$

In the case of confining theories we have $g_{t t}\left(r_{0}\right)>0$, so we can satisfy this equation for any finite $g_{\phi \phi}\left(r_{0}\right)$ by adjusting $\mu$.

Since the geodesic equation is second order, the acceleration, $\ddot{r}$, must also vanish for our geodesic to remain at a fixed value of $r$. Looking at the equations of motion (2.2) we see that such a condition implies

$$
\partial_{r}\left(g_{t t}\right)=\frac{E^{2}}{\mu^{2}} \partial_{r} g_{\phi \phi}
$$


For confining theories, there is a minimum $r=r_{0}$ for which $\partial_{r} g_{t t}\left(r_{0}\right)=0$. If we assume that $g_{\phi \phi}$ depends smoothly on $r$, and only through the magnitude $\left|r-r_{0}\right|$, then Eq. (2.5) is also easily satisfied.

It is interesting to note that the conditions (2.4) and (2.5) derived here look very similar to the ones that appear to describe confining theories in [16] (derived from considering Wilson loops) except that the metric component $g_{\phi \phi}$ now plays an important role. This role comes from looking at charged Wilson loops, as we shall see in section 7.

To summarize, we are interested in looking at objects of large charge in confining theories. We know of several such confining theories which have dual SUGRA descriptions when embedded in useful UV theories. It becomes easy to characterize the objects we want, regardless of the vagaries of their UV completion, if we look at the states localized near a null geodesic at the minimum radius (confinement scale). As we have shown, such null geodesics exist under very generic conditions for confining backgrounds.

\section{The Maldacena-Núñez background}

We begin by finding an appropriate null geodesic at $r=r_{0}$ in the MN case. ${ }^{2}$ This case is technically easier to carry out, although it turns out to be more difficult to connect with the dual field theory, due to a number of complicating features. It should be viewed, then, as a technical warm-up exercise; we do not have a full understanding of its properties.

The MN background whose IR regime is associated with $\mathcal{N}=1 \mathrm{SYM}$ theory is that of a large number of D5 branes wrapping an $S^{2}$. To be more precise: (i) the dual field theory to this SUGRA background is the $\mathcal{N}=1 \mathrm{SYM}$ contaminated with KK modes which cannot be de-coupled from the IR dynamics, (ii) the IR regime is described by the SUGRA in the vicinity of the origin where the $S^{2}$ shrinks to zero size.

The full MN SUGRA background includes the metric, the dilaton and the RR three-form. In [13] an explicit expression of the background was written down based on S-dualizing the background of large N wrapped NS5 branes. The latter solution was constructed by uplifting to ten dimensions an $S U(2)$ seven dimensional gauged SUGRA for which the spin connection of the $S^{2}$ is identified with the $U(1) \in S U(2)$ gauge connection [20].

The background takes the following form

$$
\begin{aligned}
d s_{s t r}^{2} & =e^{\phi_{D}}\left[d x_{\mu} d x^{\mu}+\alpha^{\prime} g_{s} N\left(d \rho^{2}+e^{2 g(\rho)}\left(d \theta_{1}^{2}+\sin ^{2} \theta_{1} d \phi_{1}^{2}\right)+\frac{1}{4} \sum_{a}\left(w^{a}-A^{a}\right)^{2}\right)\right] \\
e^{2 \phi_{D}} & =e^{2 \phi_{D}, 0} \frac{\sinh 2 \rho}{2 e^{g(\rho)}} \\
H^{R R} & =g_{s} N\left[-\frac{1}{4}\left(w^{1}-A^{1}\right) \wedge\left(w^{2}-A^{2}\right) \wedge\left(w^{3}-A^{3}\right)+\frac{1}{4} \sum_{a} F^{a} \wedge\left(w^{a}-A^{a}\right)\right]
\end{aligned}
$$

where $\mu=0,1,2,3$, we set the integration constant $e^{\phi_{D_{0}}}=\sqrt{g_{s} N}$. The expressions for $e^{2 g(\rho)}$

\footnotetext{
${ }^{2} \mathrm{~A}$ certain Penrose limit of the MN solution was discussed in [6].
} 
and the gauge field $A$ are given by

$$
\begin{aligned}
e^{2 g} & =\rho \operatorname{coth} 2 \rho-\frac{\rho^{2}}{\sinh ^{2} 2 \rho}-\frac{1}{4} \\
A & =\frac{1}{2}\left[\sigma^{1} a(\rho) d \theta_{1}+\sigma^{2} a(\rho) \sin \theta_{1} d \phi_{1}+\sigma^{3} \cos \theta_{1} d \phi_{1}\right] \\
a(\rho) & =\frac{2 \rho}{\sinh 2 \rho}
\end{aligned}
$$

and the one-forms $w^{a}$ are given by:

$$
\begin{aligned}
\frac{i}{2} w^{a} \sigma^{a} & =d g g^{-1} \\
w^{1}+i w^{2} & =e^{-i \psi}\left(d \theta_{2}+i \sin \theta_{2} d \phi_{2}\right), \\
g & =e^{\frac{i \psi \sigma^{3}}{2}} e^{\frac{i \theta_{2} \sigma^{1}}{2}} e^{\frac{i \phi_{2} \sigma^{3}}{2}}
\end{aligned}
$$

Note that we use notation where $x^{0}, x^{i}$ have dimension of length whereas $\rho$ and the angles $\theta_{1}, \phi_{1}, \theta_{2}, \phi_{2}, \psi$ are dimensionless and hence the appearance of the $\alpha^{\prime}$ in front of the transverse part of the metric. Moreover, following the notation of [21] a factor of $g_{s} N$ is multiplying the $\alpha^{\prime}$ instead of $N$ that appears in [13].

There are several scales associated with the $\mathcal{N}=1 \mathrm{SYM}$ dual of the MN background. the string tension, the glueball masses, the KK masses and the domain wall tension. These masses are all expressed in terms of the only scale of the background, $\alpha^{\prime}$, and they take the explicit form $[13,21]$

$$
M_{g b}^{2} \sim M_{K K}^{2} \sim \frac{1}{g_{s} N \alpha^{\prime}}, \quad T_{s} \propto M_{g b}^{2}\left(g_{s} N\right)^{\frac{3}{2}}
$$

\subsection{The Penrose limit}

We would like to take a Penrose limit for this background following the general construction of section 2, namely, based on a null geodesic with $\rho=\rho_{0}$. In the metric (3.1) we can clearly see that $g_{t t}$ has a minimum at $\rho=0$. Here, the internal space in (3.1) is an $S^{3}$; this suggest that motion at $\rho=0$ along an $S^{3}$ equator gives a candidate for a null geodesic. We would like to solve for this null geodesic using a simplified metric of the form (2.1). In order to do this, we must switch to a coordinate system where motion along the $S^{3}$ equator is parameterized by a single angle, $\phi_{+}$, and such that the description of the geodesic's neighborhood is particularly simple. Specifically we will ensure that any dependence of the metric on the distance away from the chosen $S^{3}$ equator has to be at least quadratic. This will guarantee that we can set the first and second derivatives of any deviation to zero in the equation of motion and solve for the null geodesic in terms of just the variables $t, \rho$ and $\phi_{+}$.

The coordinate system above is hard to find if we start from the explicit form of the metric in (3.1). There exists, however, a simpler approach using the fact that at the origin the gauge field (3.4) is pure gauge, namely,

$$
i A=d h h^{-1}+O\left(\rho^{2}\right) \quad \text { with } \quad h=e^{i \sigma^{1} \theta_{1} / 2} e^{i \sigma^{3} \phi_{1} / 2} .
$$


Performing a gauge transformation $A \rightarrow h^{-1} A h+i h^{-1} d h$ sets the gauge field at the origin to zero up to $O\left(\rho^{2}\right)$ corrections:

$$
\begin{aligned}
A= & \left(-\frac{1}{3} \rho^{2}+\mathcal{O}\left(\rho^{4}\right)\right)\left[\sigma^{1}\left(\cos \phi_{1} d \theta_{1}-\cos \theta_{1} \sin \theta_{1} \sin \phi_{1} d \phi_{1}\right)\right. \\
& \left.+\sigma^{2}\left(\sin \phi_{1} d \theta_{1}+\cos \theta_{1} \sin \theta_{1} \cos \phi_{1} d \phi_{1}\right)+\sigma^{3}\left(\sin ^{2} \theta_{1} d \phi_{1}\right)\right] .
\end{aligned}
$$

Note that this is just

$$
A=-\frac{1}{3}\left[\left(r_{2}^{2} d \alpha_{2}\right) \sigma^{1}+\left(r_{1}^{2} d \alpha_{1}\right) \sigma^{2}+\left(r_{3}^{2} d \alpha_{3}\right) \sigma^{3}\right]
$$

where $\left(r_{a}, \alpha_{a}\right)$ are the polar coordinates for the plane inside $R^{3} \sim R \times S^{2}$ which is perpendicular to the $x^{a}$ axis. It is straightforward to see that if we boost along a direction $\omega^{a}$ on the $S^{3}$, the $A^{a}$ component of (3.14) will give the only correction to the Penrose limit, proportional to $r_{a}^{2} d \alpha_{a} d x^{+}$. For example, if we choose to boost along the great circle on $S^{3}$ defined by $\theta_{2}=0$ and $\phi_{2}=\psi$ (hence boosting along $\omega^{3}$ ) and make the following change of variables

$$
\begin{aligned}
& d t=d x^{0}, \quad x^{i} \rightarrow \frac{1}{L} x^{i}, \quad \rho=\frac{m_{0}}{L} r, \\
& \theta_{2}=\frac{2 m_{0}}{L} v, \quad \phi_{+}=\frac{1}{2}\left(\psi+\phi_{2}\right),
\end{aligned}
$$

where $L^{2}=\sqrt{g_{s} N}$ and $m_{0}=\frac{1}{\sqrt{g_{s} N \alpha^{\prime}}}$ is the glueball mass, we get a limit for the metric (3.1) of the form:

$$
\begin{aligned}
d s^{2}= & -L^{2} d t^{2}+d x_{i} d x^{i}+d r^{2}+r^{2}\left(d \theta_{1}^{2}+\sin \theta_{1}^{2} d \phi_{1}^{2}\right) \\
& +\left(d v^{2}+v^{2} d \phi_{2}^{2}\right)+\frac{L^{2}}{m_{0}^{2}} d \phi_{+}^{2}-2 v^{2} d \phi_{2} d \phi_{+}+\frac{2}{3} r^{2} \sin \theta_{1}^{2} d \phi_{1} d \phi_{+}+\mathcal{O}\left(L^{-2}\right)
\end{aligned}
$$

where the new variables $r, v$ have dimension of length. It is easy to see that boosting along $\omega^{1}$ and $\omega^{2}$ for $\theta_{2}=\frac{\pi}{2}$ and $\psi=0$ or $\frac{\pi}{2}$ will give the same type of result. Since this form of the metric is invariant under $x \rightarrow-x, x=0$ is a solution of the equation of motion. To eliminate some of the "magnetic" terms we now introduce a shift in the angles $\phi_{1}$ and $\phi_{2}$

$$
\hat{\phi}_{1}=\phi_{1}+\frac{1}{3} \phi_{+} \quad \hat{\phi}_{2}=\phi_{2}-\phi_{+}
$$

Expressed in terms of the these shifted angles the metric takes the form

$$
\begin{aligned}
d s^{2}= & L^{2}\left[-d t^{2}+\frac{1}{m_{0}^{2}} d \phi_{+}^{2}\right]+d x_{i} d x^{i} \\
& +d r^{2}+r^{2}\left(d \theta_{1}^{2}+\sin \theta_{1}^{2} d \hat{\phi}_{1}^{2}\right) \\
& +\left(d v^{2}+v^{2} d \hat{\phi}_{2}^{2}\right)-\left(v^{2}+\frac{r^{2}}{9} \sin ^{2} \theta_{1}\right) d \phi_{+}^{2}+\mathcal{O}\left(L^{-2}\right)
\end{aligned}
$$


Finally we let

$$
x^{+}=t, \quad x^{-}=\frac{L^{2}}{2}\left(t-\frac{1}{m_{0}} \phi_{+}\right),
$$

and denote the Cartesian coordinates of the $R^{3}$ associated with $d r^{2}+r^{2}\left(d \theta_{1}^{2}+\sin \theta_{1}^{2} d \hat{\phi}_{1}^{2}\right)$ as $d u_{1}^{2}+d u_{2}^{2}+d z^{2}$ (and similarly for the $v$ plane $d v^{2}+v^{2} d \hat{\phi}_{2}^{2}=d v_{1}^{2}+d v_{2}^{2}$ ). Then we take the Penrose limit $L \rightarrow \infty$ while keeping $m_{0}$ fixed, obtaining

$$
d s^{2}=-2 d x^{+} d x^{-}-m_{0}^{2}\left(\frac{1}{9} u_{1}^{2}+\frac{1}{9} u_{2}^{2}+v^{2}\right)\left(d x^{+}\right)^{2}+d \vec{x}^{2}+d \vec{z}^{2}+d u_{1}^{2}+d u_{2}^{2}+d v_{1}^{2}+d v_{2}^{2} .
$$

We thus obtain a plane wave metric with 4 massless direction (three $x$ 's and $z$ ), two directions $(v)$ with mass $m_{0}$ and two directions $(u)$ with mass $\frac{1}{3} m_{0}$.

Next we would like to consider the Penrose limit of the three form field strength $H^{R R}$. According to the procedure of Güven [9] since $H^{R R}$ is the field strength of the 2-form $A_{2}$, the $L \rightarrow \infty$ limit takes the form $H^{R R}=L^{2} \tilde{H}^{R R}$. The only terms that survive this limit are $w^{1} \wedge w^{2} \wedge w^{3}$ and $F^{3} \wedge w^{3}$. All the other terms are suppressed by factors of $1 / L$. The final expression is

$$
H^{R R}=-2 m_{0} d x^{+} \wedge\left[d v_{1} \wedge d v_{2}+1 / 3 d z_{1} \wedge d z_{2}\right] .
$$

As expected the only non-trivial components are of the form $H_{+i j}$. As a consistency check we examine the equation of motion

$$
R_{++}=\frac{1}{4}\left(H_{+i j} H_{+}^{i j}-\frac{1}{12} g_{++} H_{i j k} H^{i j k}\right)
$$

The component $R_{++}$of the Ricci tensor associated with the metric $(3.20)$ is $R_{++}=\sum_{i} m_{i}^{2}=$ $(20 / 9) m_{0}^{2}$. It is easy to see that by substituting (3.21) into the equation of motion we get exactly the same expression also in the right hand side of the equation. Notice that the second term in this side of the equation vanishes since the terms of the 3 -form have a structure of $H_{+i j}$ and $g^{++}=0$.

The Hamiltonian is:

$$
H=-p_{+}=i \partial_{+}=E-m_{0}\left(-\frac{1}{3} J_{1}+J_{2}+J_{\psi}\right) \equiv E-m_{0} J
$$

and the momentum $P^{+}$is

$$
P^{+}=-\frac{1}{2} p_{-}=\frac{i}{2} \partial_{-}=\frac{m_{0}}{L^{2}}\left(-\frac{1}{3} J_{1}+J_{2}+J_{\psi}\right)=m_{0} \frac{J}{\sqrt{g_{s} N}} .
$$

where $J_{1}, J_{2}$ and $J_{\psi}$ denote $-i \partial_{\phi_{1}},-i \partial_{\phi_{2}}$ and $-i \partial_{\psi}$ respectively.

Here we see something non-trivial, and slightly distressing, about this plane-wave limit. The metric (3.1) with the new gauge field (3.13) contains only two global $U(1)$ isometries, $U(1)_{L}=J_{2}$ and $U(1)_{R}=J_{\psi}-J_{1}$ (modulo $S U(2)$ rotations). Therefore the symmetry current $-\frac{1}{3} J_{1}+J_{2}+J_{\psi}$ does not represent an isometry of the full MN solution; it is an accidental symmetry arising only in the Penrose limit. It therefore governs the hadrons of the gauge theory only in the large-charge limit. Moreover, since this accidental symmetry does not commute with 
supersymmetry, the corresponding string theory will exhibit the supersymmetry of the gauge theory in a slightly unexpected fashion.

We could choose to define $\hat{\phi}_{1}=\phi_{1}+\phi_{+}$so that $J$ would now be in the diagonal of $U(1)_{L}$ and $U(1)_{R}$. Unfortunately, this leaves us with a so-called "magnetic" term of the form $d \phi_{1} d \phi_{+}$ in the metric. Solving the string theory in this background is slightly more complicated, but at the end boils down to shifting the whole spectrum of energies derived from the original background by $\frac{2}{3} m_{0} J_{1}$.

Perhaps one way to understand this strange appearance of a magnetic term for what should be the natural choice of symmetry current is to look at the dual field theory of the D5-brane. If we look at the Kaluza-Klein spectrum for this D5-brane, we can quickly see that it contains massive scalar and vector multiplets. The key feature here is that the scalar multiplets transform under both of the global $S U(2)$ 's, while the vectors only transform under $S U(2)_{R}$ ! Capturing the full dynamics of the Penrose limit requires us to look at objects with scalar components from both the lowest mass scalar multiplet and the lowest mass vector multiplet. The $L \leftrightarrow R$ asymmetry of the vector scalars hints at a genesis for a magnetic term.

Fortunately, the corresponding plane-wave limit of the KS solution is not plagued with an un-natural choice of boost symmetry. Although technically more challenging to obtain, it turns out to be much more elegant and much easier to interpret.

\section{The Klebanov-Strassler background}

We begin by reviewing the KS background, which is obtained by considering a collection of $N$ regular and $M$ fractional D3-branes in the geometry of the deformed conifold [14] (see also $[15])$. The 10-d metric is of the form:

$$
d s_{10}^{2}=h^{-1 / 2}(\tau) d x_{\mu} d x^{\mu}+h^{1 / 2}(\tau) d s_{6}^{2},
$$

where $d s_{6}^{2}$ is the metric of the deformed conifold $[22,23]$ :

$d s_{6}^{2}=\frac{1}{2} \varepsilon^{4 / 3} K(\tau)\left[\frac{1}{3 K^{3}(\tau)}\left(d \tau^{2}+\left(g^{5}\right)^{2}\right)+\cosh ^{2}\left(\frac{\tau}{2}\right)\left[\left(g^{3}\right)^{2}+\left(g^{4}\right)^{2}\right]+\sinh ^{2}\left(\frac{\tau}{2}\right)\left[\left(g^{1}\right)^{2}+\left(g^{2}\right)^{2}\right]\right]$.

where

$$
K(\tau)=\frac{(\sinh (2 \tau)-2 \tau)^{1 / 3}}{2^{1 / 3} \sinh \tau}
$$

and

$$
\begin{aligned}
g^{1} & =\frac{1}{\sqrt{2}}\left[-\sin \theta_{1} d \phi_{1}-\cos \psi \sin \theta_{2} d \phi_{2}+\sin \psi d \theta_{2}\right] \\
g^{2} & =\frac{1}{\sqrt{2}}\left[d \theta_{1}-\sin \psi \sin \theta_{2} d \phi_{2}-\cos \psi d \theta_{2}\right] \\
g^{3} & =\frac{1}{\sqrt{2}}\left[-\sin \theta_{1} d \phi_{1}+\cos \psi \sin \theta_{2} d \phi_{2}-\sin \psi d \theta_{2}\right]
\end{aligned}
$$




$$
\begin{aligned}
g^{4} & =\frac{1}{\sqrt{2}}\left[d \theta_{1}+\sin \psi \sin \theta_{2} d \phi_{2}+\cos \psi d \theta_{2}\right] \\
g^{5} & =d \psi+\cos \theta_{1} d \phi_{1}+\cos \theta_{2} d \phi_{2} .
\end{aligned}
$$

The 3-form fields are:

$$
\begin{gathered}
F_{3}=\frac{M \alpha^{\prime}}{2}\left\{g^{5} \wedge g^{3} \wedge g^{4}+d\left[F(\tau)\left(g^{1} \wedge g^{3}+g^{2} \wedge g^{4}\right)\right]\right\} \\
=\frac{M \alpha^{\prime}}{2}\left\{g^{5} \wedge g^{3} \wedge g^{4}(1-F)+g^{5} \wedge g^{1} \wedge g^{2} F\right. \\
\left.+F^{\prime} d \tau \wedge\left(g^{1} \wedge g^{3}+g^{2} \wedge g^{4}\right)\right\}
\end{gathered}
$$

and

$$
\begin{gathered}
B_{2}=\frac{g_{s} M \alpha^{\prime}}{2}\left[f(\tau) g^{1} \wedge g^{2}+k(\tau) g^{3} \wedge g^{4}\right] \\
H_{3}=d B_{2}=\frac{g_{s} M \alpha^{\prime}}{2}\left[d \tau \wedge\left(f^{\prime} g^{1} \wedge g^{2}+k^{\prime} g^{3} \wedge g^{4}\right)\right. \\
\left.+\frac{1}{2}(k-f) g^{5} \wedge\left(g^{1} \wedge g^{3}+g^{2} \wedge g^{4}\right)\right] .
\end{gathered}
$$

The self-dual 5 -form field strength is decomposed as $\tilde{F}_{5}=\mathcal{F}_{5}+\star \mathcal{F}_{5}$, with

$$
\mathcal{F}_{5}=B_{2} \wedge F_{3}=\frac{g_{s} M^{2}\left(\alpha^{\prime}\right)^{2}}{4} \ell(\tau) g^{1} \wedge g^{2} \wedge g^{3} \wedge g^{4} \wedge g^{5}
$$

where

$$
\ell=f(1-F)+k F
$$

and

$$
\star \mathcal{F}_{5}=4 g_{s} M^{2}\left(\alpha^{\prime}\right)^{2} \varepsilon^{-8 / 3} d x^{0} \wedge d x^{1} \wedge d x^{2} \wedge d x^{3} \wedge d \tau \frac{\ell(\tau)}{K^{2} h^{2} \sinh ^{2}(\tau)} .
$$

The functions introduced in defining the form fields are:

$$
\begin{aligned}
F(\tau) & =\frac{\sinh \tau-\tau}{2 \sinh \tau}, \\
f(\tau) & =\frac{\tau \operatorname{coth} \tau-1}{2 \sinh \tau}(\cosh \tau-1), \\
k(\tau) & =\frac{\tau \operatorname{coth} \tau-1}{2 \sinh \tau}(\cosh \tau+1) .
\end{aligned}
$$

The equation for the warp factor is

$$
h^{\prime}=-\alpha \frac{f(1-F)+k F}{K^{2}(\tau) \sinh ^{2} \tau},
$$

where

$$
\alpha=4\left(g_{s} M \alpha^{\prime}\right)^{2} \varepsilon^{-8 / 3} .
$$


For large $\tau$ we impose the boundary condition that $h$ vanishes. The resulting integral expression for $h$ is

$$
h(\tau)=\alpha \frac{2^{2 / 3}}{4} I(\tau)=\left(g_{s} M \alpha^{\prime}\right)^{2} 2^{2 / 3} \varepsilon^{-8 / 3} I(\tau)
$$

where

$$
I(\tau) \equiv \int_{\tau}^{\infty} d x \frac{x \operatorname{coth} x-1}{\sinh ^{2} x}(\sinh (2 x)-2 x)^{1 / 3} .
$$

The above integral has the following expansion in the IR:

$$
I(\tau \rightarrow 0) \rightarrow a_{0}-a_{1} \tau^{2}+\mathcal{O}\left(\tau^{4}\right)
$$

where $a_{0} \approx 0.71805$ and $a_{1}=2^{2 / 3} 3^{2 / 3} / 18$. The absence of a linear term in $\tau$ reassures us that we are really expanding around the end of space, where the Wilson loop will find it more favorable to arrange itself.

\subsection{A convenient parameterization of the KS background}

As it turns out, the above parameterization of the metric will not be quite suitable for our purposes since the 1 -forms $d \psi, d \theta_{i}$ and $d \phi_{i}$ mix the 1 -forms of the $S^{3}$ at the origin, $g^{3}, g^{4}$ and $g^{5}$, with those from the $S^{2}, g^{1}$ and $g^{2}$. In these coordinates it would be problematic to try and get a Penrose limit by boosting along $g^{5}$. For example, boosting along $g^{5}$ by shifting $\psi$ does not work as this coordinate is Hopf-fibered over the $S^{2}$ which shrinks to zero size at the origin.

Instead, we pick explicitly separate coordinates ${ }^{3}$ for the $S^{3}$ and the $S^{2}$ (see the appendix for more detail) : for the $S^{3}$ an $S U(2)$ matrix

$$
T=e^{\frac{i}{2} \phi^{\prime} \sigma_{3}} e^{\frac{i}{2} \theta^{\prime} \sigma_{1}} e^{\frac{i}{2} \psi^{\prime} \sigma_{3}},
$$

and for the $S^{2}$ a matrix

$$
S=e^{\frac{i}{2} \phi \sigma_{3}} e^{-\frac{i}{2} \theta \sigma_{1}} .
$$

We can now work with the 1 -forms

$$
T^{\dagger} d T=-d T^{\dagger} T=\frac{i}{2} \omega^{a} \sigma_{a}
$$

and

$$
d \theta, \sin \theta d \phi .
$$

We now re-write the metric for the deformed conifold in terms of these 1 -forms as

$$
\begin{aligned}
\epsilon^{-\frac{4}{3}} d s_{6}^{2}= & \frac{1}{4} K(\tau) \cosh (\tau)\left(d \tau^{2}+\left(\omega^{a}\right)^{2}\right) \\
+ & K(\tau) \sinh ^{2}\left(\frac{\tau}{2}\right)\left[\left(d \theta^{2}+\sin ^{2} \theta d \phi^{2}\right)\right. \\
& -\left(\sin \phi \omega^{1}+\cos \phi \omega^{2}\right)(d \theta)
\end{aligned}
$$

\footnotetext{
${ }^{3}$ This is very similar in spirit to the gauge transformation we used in the last section.
} 


$$
\begin{gathered}
\left.-\left(\cos \theta \cos \phi \omega^{1}-\cos \theta \sin \phi \omega^{2}-\sin \theta \omega^{3}\right)(\sin \theta d \phi)\right] \\
+\frac{1}{4} K^{\prime}(\tau) \sinh (\tau)\left[d \tau^{2}+\left(\sin \theta \cos \phi \omega^{1}+\sin \theta \sin \phi \omega^{2}+\cos \theta \omega^{3}\right)^{2}\right] .
\end{gathered}
$$

\subsection{Plane wave limit}

Due to the behavior of the warp factor in the IR $(h \rightarrow$ constant), it is clear that in the deep IR there are null geodesics that lie at $\tau=0$. Hence, we will consider a Penrose limit where we expand around $\tau=0$, in a manner similar to the BMN expansion near the center of $A d S$ in global coordinates. (The analogy here is purely formal, however, as the physical meaning of the time variable in global versus Poincaré coordinates is very different in the field theory dual.) An important guide in taking the limit that we want is that we should keep finite the mass of the glueball

$$
M_{g b} \propto \frac{\varepsilon^{\frac{2}{3}}}{g_{s} M \alpha^{\prime}} .
$$

Note that now the dynamics in KS are such that the flux tube tension is

$$
T_{s} \propto M_{g b}^{2}\left(g_{s} M\right) .
$$

We start the machinery for the Penrose limit by expanding the KS metric up to quadratic terms in $\tau / L$, and eventually taking $L \rightarrow \infty$. We also want to take a Penrose limit near an equator on the $S^{3}$ at the origin. Without loss of generality, we can choose coordinates $\theta^{\prime}, \phi^{\prime}$ and $\psi^{\prime}$ such that this equator sits at $\theta^{\prime}=0$ and is generated by $\phi^{\prime}+\psi^{\prime}$ (to first order this is $\omega^{3}$ ). To take the limit, we will need also need to re-scale the coordinate $\theta^{\prime} \rightarrow \theta^{\prime} / L$, i.e., the 1 -forms $\omega^{1}, \omega^{2}$ will go like $\frac{1}{L}$. This scaling simplifies the deformed conifold metric (4.21):

$$
\begin{aligned}
L^{2} d s_{6}^{2} & =\frac{\epsilon^{\frac{4}{3}}}{2^{\frac{5}{3}} 3^{\frac{1}{3}}}\left[d \tau^{2}+\left(d \theta^{\prime}\right)^{2}+\left(\theta^{\prime}\right)^{2}\left(d \phi^{\prime 2}\right)+L^{2}\left(\omega^{3}\right)^{2}+\frac{2}{5} \tau^{2}\left(\omega^{3}\right)^{2}\right. \\
& \left.+\tau^{2} d \Omega_{2}^{2}+\left(\tau^{2} \sin ^{2} \theta\right) d \phi \omega^{3}-\frac{1}{5} \tau^{2} \cos ^{2} \theta\left(\omega^{3}\right)^{2}\right]
\end{aligned}
$$

If we expand $\omega_{3}^{2}$ as

$$
\omega_{3}^{2}=4\left(d \phi_{+}\right)^{2}-2\left(\frac{\theta^{\prime}}{L}\right)^{2} d \phi^{\prime} d \phi_{+},
$$

we can write the full 10-dimensional metric in the limit as:

$$
\begin{aligned}
d s_{10}^{2} & =-\frac{c_{0}^{2}}{L^{2}}\left[L^{2}+\frac{a_{1}}{2 a_{0}} \tau^{2}\right] d t^{2}+c_{0}^{2} \delta_{i j} d x^{i} d x^{j} \\
& +\frac{c_{1}^{2}}{L^{2}}\left[4 L^{2} d \phi_{+}^{2}+d \tau^{2}+\tau^{2}\left(d \theta^{2}+\sin ^{2} \theta d \phi^{2}\right)+\left(d \theta^{\prime}\right)^{2}+\left(\theta^{\prime}\right)^{2}\left(d \phi^{\prime}\right)^{2}\right. \\
& +2 \tau^{2} \sin ^{2} \theta d \phi d \phi_{+}-2 \theta^{2} d \phi^{\prime} d \phi_{+} \\
& \left.+4 \tau^{2} \sin ^{2} \theta\left(\frac{2}{5}-\frac{a_{1}}{2 a_{0}}\right)\left(d \phi_{+}\right)^{2}+4 \tau^{2} \cos ^{2} \theta\left(\frac{1}{5}-\frac{a_{1}}{2 a_{0}}\right)\left(d \phi_{+}\right)^{2}\right]
\end{aligned}
$$


with

$$
c_{0}^{2}=\frac{\varepsilon^{4 / 3}}{2^{1 / 3} g_{s} M \alpha^{\prime} a_{0}^{1 / 2}}, \quad c_{1}^{2}=\frac{g_{s} M \alpha^{\prime} a_{0}^{1 / 2}}{2^{4 / 3} 3^{1 / 3}} .
$$

As a next step, we know that the Penrose limit calls for the overall metric to be re-scaled by $L^{2}$. We can accomplish this by taking

$$
c_{0} \rightarrow \infty, \quad c_{1} \rightarrow \infty
$$

while keeping constant

$$
\frac{c_{0}}{L}=1, \quad \frac{c_{0}}{c_{1}}=\frac{\varepsilon^{\frac{2}{3}}}{g_{s} M \alpha^{\prime}}\left(\frac{24}{a_{0}^{3}}\right)^{\frac{1}{6}}=2 m_{0} .
$$

With these scalings in mind, we make the following further changes in coordinates in order to take the Penrose limit:

$$
\phi_{+}=\frac{1}{2}\left(\phi^{\prime}+\psi^{\prime}\right), \quad x^{+}=t, \quad x^{-}=\frac{c_{0}^{2}}{2}\left(t-\frac{2 c_{1}}{c_{0}} \phi_{+}\right),
$$

with

$$
\begin{aligned}
x^{i} \rightarrow \frac{x^{i}}{L}, & \varphi=\frac{1}{2}\left(\phi^{\prime}-\psi^{\prime}\right), \quad v=\frac{c_{1}}{c_{0}} \theta^{\prime} e^{i \varphi}, \\
z=\frac{c_{1}}{c_{0}} \tau \cos \theta & \tilde{\phi}=\phi+\phi_{+}, \quad u=\frac{c_{1}}{c_{0}} \tau \sin \theta e^{i \tilde{\phi}} .
\end{aligned}
$$

After we take $L \rightarrow \infty$ the resulting metric is

$$
\begin{aligned}
d s^{2}=-4 d x^{+} d x^{-} & -m_{0}^{2}\left[\left(\frac{4 a_{1}}{a_{0}}-\frac{4}{5}\right) z^{2}+\left(\frac{4 a_{1}}{a_{0}}-\frac{3}{5}\right) u \bar{u}+v \bar{v}\right]\left(d x^{+}\right)^{2} \\
& +d x^{i} d x_{i}+d z^{2}+d u d \bar{u}+d v d \bar{v} .
\end{aligned}
$$

\subsection{The various forms in the plane wave limit}

We now turn to the construction of the forms in the new coordinates. A convenient relation we will use in what follows is:

$$
\frac{g_{s} M \alpha^{\prime}}{L^{2}} m_{0}^{2}=a_{0}^{-\frac{1}{2}} 2^{\frac{4}{3}} 3^{\frac{1}{3}} \frac{c_{1}^{2}}{c_{0}^{2}} m_{0}^{2}=a_{0}^{-\frac{1}{2}} 2^{-\frac{2}{3}} 3^{\frac{1}{3}}=\left(\frac{a_{1}}{a_{0}}\right)^{\frac{1}{2}} \frac{3}{\sqrt{2}} .
$$

The earlier expressions for the $g^{i}$ s (4.4) allow us to write down the Ramond-Ramond 3form:

$$
\begin{aligned}
F_{3} & =\frac{M \alpha^{\prime}}{2}\left\{g^{5} \wedge g^{3} \wedge g^{4}+d\left[F(\tau)\left(g^{1} \wedge g^{3}+g^{2} \wedge g^{4}\right)\right]\right\} \\
& \rightarrow \frac{3 i m_{0}}{\sqrt{2} g_{s}}\left(\frac{a_{1}}{a_{0}}\right)^{\frac{1}{2}} d x^{+} \wedge\left(\frac{1}{3} d u \wedge d \bar{u}+d v \wedge d \bar{v}\right)
\end{aligned}
$$


Similarly we write down the NS-NS 2-form as:

$$
\begin{aligned}
B_{2} & =\frac{g_{s} M \alpha^{\prime}}{2}\left[f(\tau) g^{1} \wedge g^{2}+k(\tau) g^{3} \wedge g^{4}\right] \\
& \rightarrow \frac{m_{0}}{\sqrt{2}}\left(\frac{a_{1}}{a_{0}}\right)^{\frac{1}{2}} d x^{+} \wedge(-i)[u d \bar{v}-\bar{u} d v]
\end{aligned}
$$

The complex 3-form field strength obtained by combining the above forms is:

$$
\begin{aligned}
G_{3} & =H_{3}+i g_{s} F_{3} \\
& =\frac{m_{0}}{\sqrt{2}}\left(\frac{a_{1}}{a_{0}}\right)^{\frac{1}{2}} d x^{+} \wedge[(d u \wedge d \bar{u}+3 d v \wedge d \bar{v})+i(d u \wedge d \bar{v}-d \bar{u} \wedge d v)]
\end{aligned}
$$

which has as a norm

$$
\left(G_{3}\right)_{+i j}\left(\bar{G}_{3}\right)_{+}^{i j}=48 \frac{a_{1}}{a_{0}} m_{0}^{2}
$$

As an extra check we verify that the only nontrivial equation of motion

$$
R_{++}=\frac{1}{4}\left(G_{3}\right)_{+i j}\left(\bar{G}_{3}\right)_{+}^{i j}
$$

is satisfied. Indeed from (4.32) we obtain

$$
R_{++}=m_{0}^{2}\left[\left(\frac{4 a_{1}}{a_{0}}-\frac{4}{5}\right)+2\left(\frac{4 a_{1}}{a_{0}}-\frac{3}{5}\right)+2\right]=12 \frac{a_{1}}{a_{0}} m_{0}^{2},
$$

which matches perfectly with the 3 -form.

\subsection{Operators and symmetries}

The Hamiltonian now takes the form

$$
\begin{aligned}
H=-p_{+} & =i \partial_{+}=i\left[\partial_{t}+m_{0}\left(\partial_{\phi^{\prime}}+\partial_{\psi^{\prime}}-\partial_{\phi}\right)\right] \\
& =E-m_{0} J
\end{aligned}
$$

with

$$
\begin{aligned}
P^{+} & =\frac{i}{2} \partial_{-}=-\frac{i}{c_{0}^{2}} m_{0}\left(\partial_{\phi^{\prime}}+\partial_{\psi^{\prime}}-\partial_{\phi}\right) \\
& =m_{0}\left(\frac{J}{c_{0}^{2}}\right) .
\end{aligned}
$$

The geodesic used in our Penrose limit is generated by a symmetry which we will call $U(1)_{D}$. Its action on the matrices $T$ and $S$ is

$$
\begin{aligned}
e^{i \alpha J}: T & \rightarrow e^{i \frac{\alpha}{2} \sigma_{3}} T e^{i \frac{\alpha}{2} \sigma_{3}} \\
S & \rightarrow e^{-i \frac{\alpha}{2} \sigma_{3}} S
\end{aligned}
$$


which means its action on our general complex coordinate matrix for the conifold is $W=$ $T S W_{\epsilon} \sigma_{3} S^{\dagger} \sigma_{3}$, is:

$$
e^{i \alpha J}: W \rightarrow e^{i \frac{\alpha}{2} \sigma_{3}} W e^{i \frac{\alpha}{2} \sigma_{3}} .
$$

The geodesic is left invariant by an orthogonal abelian symmetry acting on $W$, which we will term $U(1)_{A}$, with

$$
e^{i \alpha J_{A}}: W \rightarrow e^{i \frac{\alpha}{2} \sigma_{3}} W e^{-i \frac{\alpha}{2} \sigma_{3}} .
$$

In this case,

$$
J_{A}=-i\left(\partial_{\phi^{\prime}}-\partial_{\psi^{\prime}}+\partial_{\phi}\right) .
$$

One may check that both $u$ and $v$ carry charge 1 under $J_{A}$, while $x^{+}$and $z$ are neutral, and that the metric and 3 -forms are also neutral.

Thus, in contrast to our results in the MN case, the symmetry current corresponding to the charge $J$ is conserved by the full gauge theory, and corresponds to an isometry of the full KS metric. Moreover, it commutes with the supersymmetry of the full theory. This makes the corresponding string theory, and its interpretation, reasonably straightforward.

\section{The plane wave string and its Hamiltonian}

In this section we work out the string Hamiltonian for the KS and MN plane waves, keeping an eye out for common distinguishing features as well as differences. We will treat the bosonic sector first, and then discuss the fermionic oscillators and the effects of supersymmetry.

\subsection{Bosonic Sector}

The form of the KS metric (4.32) directly implies that the bosonic sector of the system is described by three massless fields with frequencies $w_{n}=n$, and five massive (no zero-frequency mode) fields. Due to the presence of a B-field, four of the latter organize themselves as two sets of coupled fields (see appendix B). The frequencies for the five massive fields are

$$
\begin{aligned}
w_{n}^{z} & =\sqrt{n^{2}+\hat{m}_{z}^{2}} \\
\left(\omega_{n}^{ \pm}\right)^{2} & =\frac{1}{2}\left[2 n^{2}+\hat{m}_{v}^{2}+\hat{m}_{u}^{2} \pm \sqrt{\left(\hat{m}_{v}^{2}-\hat{m}_{u}^{2}\right)^{2}+4 n^{2} \hat{m}_{B}^{2}}\right],
\end{aligned}
$$

where

$$
\begin{aligned}
\hat{m}_{z}=\left(m_{0} p^{+} \alpha^{\prime}\right)\left(\frac{4 a_{1}}{a_{0}}-\frac{4}{5}\right)^{\frac{1}{2}}, & \hat{m}_{u}=\left(m_{0} p^{+} \alpha^{\prime}\right)\left(\frac{4 a_{1}}{a_{0}}-\frac{3}{5}\right)^{\frac{1}{2}}, \\
\hat{m}_{v}=m_{0} p^{+} \alpha^{\prime}, & \hat{m}_{B}=\sqrt{2} m_{0} p^{+} \alpha^{\prime}\left(\frac{a_{1}}{a_{0}}\right)^{1 / 2} .
\end{aligned}
$$

Glancing at the form of the frequencies for the coupled fields we notice that the presence of the B-field (parameterized by $\hat{m}_{B}$ above) only affects the frequencies for $n>0$. At zero-level the coupled fields have frequencies $\omega_{0}^{+}=\hat{m}_{v}$ and $\omega_{0}^{-}=\hat{m}_{u}$ which can naturally be identified with excitations of $v$ and $u$. 
We can obtain the structure for the lowest-lying excitations of the MN plane wave case in a similar fashion to the case above. Due to the absence of a B-field in the MN plane--wave the directions $u$ and $v$ no-longer mix, so we can recycle formula in eq. (5.1) with

$$
\hat{m}_{z}=0, \quad \hat{m}_{u}=\frac{1}{3}\left(m_{0} p^{+} \alpha^{\prime}\right), \quad \hat{m}_{v}=m_{0} p^{+} \alpha^{\prime}, \quad \hat{m}_{B}=0 .
$$

and rename

$$
\omega_{n}^{+}=\omega_{n}^{v}, \quad \omega_{n}^{-}=\omega_{n}^{u} .
$$

What do the MN and KS spectrum have in common? First, they have an identical stringy sector for the three directions which correspond to the three spatial directions in the dual gauge theory. Second, they have the same massive level-zero modes in two of the internal directions $\left(v\right.$ and $\bar{v}$ ). These come from a natural $S^{3}$ structure in both the MN and KS cases; they represent the direction in this sphere normal to the reference geodesic. Even though this common feature is ruined by the $\mathrm{B}$-field for more excited states, we will refer to oscillation in $v$ as the "universal sector." The remaining oscillation directions are less universal and come from the combination of the radial direction and the $S^{2}$-like structure. In particular, what is striking here is that $\hat{m}_{z}$ is zero in the MN case, while all the $\hat{m}$ 's are positive in the KS case. In either case, it is interesting to note that all the quantities in this "non-universal sector" are smaller than $\hat{m}_{v}$ since $\sqrt{\frac{4 a_{1}}{a_{0}}-\frac{4}{5}} \approx .47$ and $\sqrt{\frac{4 a_{1}}{a_{0}}-\frac{3}{5}} \approx .65$.

The whole bosonic Hamiltonian can be written explicitly following standard manipulations. Here we provide the needed notation to understand its form; the details are given in appendix B. We define number operators

$$
\begin{array}{ll}
\mathcal{N}_{R}=\sum_{n=1}^{\infty} n\left(a_{n}^{i \dagger} a_{n}^{i}\right), & \mathcal{N}_{L}=\sum_{n=1}^{\infty} n\left(\tilde{a}_{n}^{i \dagger} \tilde{a}_{n}^{i}\right) \\
N_{R}=\sum_{n=1}^{\infty} n\left(a_{n}^{s \dagger} a_{n}^{s}\right), & N_{L}=\sum_{n=1}^{\infty} n\left(\tilde{a}_{n}^{s \dagger} \tilde{a}_{n}^{s}\right)
\end{array}
$$

and sub-Hamiltonians

$$
\begin{aligned}
& H_{0}=w_{0}^{s}\left(a_{0}^{s \dagger} a_{0}^{s}\right), \\
& H_{R}=\sum_{n=1}^{\infty} w_{n}^{s}\left(a_{n}^{s \dagger} a_{n}^{s}\right), \quad H_{L}=\sum_{n=1}^{\infty} w_{n}^{s}\left(\tilde{a}_{n}^{s \dagger} \tilde{a}_{n}^{s}\right) .
\end{aligned}
$$

The subindex $i=1,2,3$ refers to the three flat directions in the plane wave (spatial directions in the gauge theory), while the index $s=4,5,6,7,8$ runs over the internal directions. There is implied summation over the indices $i$ and $s$. The full bosonic light-cone Hamiltonian is

$$
\begin{aligned}
H=-P^{-} & =H_{\|}+H_{\perp} \\
& =\left[\frac{P_{i}^{2}}{2 P^{+}}+\frac{1}{2 \alpha^{\prime} P^{+}}\left(\mathcal{N}_{R}+\mathcal{N}_{L}\right)\right]+\left[\frac{1}{2 \alpha^{\prime} P^{+}}\left(H_{0}+H_{R}+H_{L}\right)\right] .
\end{aligned}
$$

The Hamiltonian is thus constructed of a contribution from the momentum and stringy excitations in the spatial directions of the field theory (index $i$ ), $H_{\|}$, and a contribution from the massive "zero" modes and excitations of the internal directions (index $s$ ), $H_{\perp}$. 
From this we may observe two important features which both the bosonic MN and KS Hamiltonians share. First, both theories have the same $H_{\|}$. Second, they have the same $\hat{m}_{v}=p^{+} \alpha^{\prime} m_{0}$. More precisely, note from (3.23) and (4.40) that we have defined $m_{0}$ in each case so that the energy $E$ of the string theory vacuum state is $J m_{0}$. The two theories then share the fact that the lowest-lying mode of $v$ shifts $E$ by exactly $m_{0}$. We will see in a moment why these features are universal.

\subsection{The fermionic sector}

Before we describe the fermionic contribution to the string spectrum in our plane waves, let us consider first what happens to the target space supersymmetries of the original solutions. Both the $\mathrm{MN}$ and $\mathrm{KS}$ background are dual to $\mathcal{N}=1$ supersymmetric field theories; their supersymmetry algebra contains exactly four supercharges. These supercharges commute with the original Hamltonian, $\partial_{t}$, and with the $S U(2)_{L} \times S U(2)_{R}$ global symmetry generators. In the Penrose limit, these supercharges are re-scaled as

$$
Q \rightarrow L \cdot Q
$$

since their Killing spinors mix with the coordinates $x^{i}$. This implies a contraction of the supersymmetry algebra

$$
\begin{aligned}
\{Q, Q\} & \propto \Gamma^{\mu} \mathcal{P}_{\mu} \\
\rightarrow \quad\{Q, Q\} & \propto \frac{1}{L^{2}}\left(\Gamma^{0}\left(i \partial_{+}+L^{2} i \partial_{-}\right)+L \Gamma^{i} i \partial_{i}\right)=\Gamma^{0} P^{+}+\mathcal{O}\left(L^{-1}\right),
\end{aligned}
$$

which tells us that the original supercharges have now become part of the 16 "kinematic" supercharges ubiquitous to pp-wave solutions $([25,27])$. This means (see [28]) that they will be non-linearly realized on the string worldsheet.

Let us now specialize to the KS case. The Hamiltonian for the KS plane-wave is shifted from the original KS Hamiltonian by a charge $\mathrm{J}$ which generates $U(1)_{D}$ in the global symmetry group. This means that it still commutes with the four supercharges above! After fixing lightcone gauge and kappa symmetry, the (non-linear) action of the kinematic supersymmetries takes the form

of multiplication by the Green-Schwarz fermionic fields $S^{\alpha}$ and $\tilde{S}^{\alpha}$. Commutation of four of the sixteen supersymmetries with the Hamiltonian then implies that two each of the eight left-moving and right-moving spinors on the worldsheet should have a zero-frequency mode. These act on the vacuum to generate a four-dimensional Hilbert space of degenerate states (two fermionic and two bosonic).

An explicit computation (see Appendix B) of the fermionic spectrum confirms this prediction. The spectrum of the string in the KS plane wave contains four fermionic fields (each of which has a left-moving and a right-moving part) with frequencies

$$
\left.\omega_{n}^{\alpha}\right|_{\alpha=1 \ldots 4}=\sqrt{n^{2}+\hat{m}_{f}^{2}}
$$

two fermionic fields with frequencies

$$
\left.\omega_{n}^{\alpha}\right|_{\alpha=5,6}=\sqrt{n^{2}+\frac{1}{2} \hat{m}_{f}^{2}+\frac{1}{2} \hat{m}_{f} \sqrt{\hat{m}_{f}^{2}+4 n^{2}}},
$$


and two fermionic fields with frequencies

$$
\left.\omega_{n}^{\alpha}\right|_{\alpha=7,8}=\sqrt{n^{2}+\frac{1}{2} \hat{m}_{f}^{2}-\frac{1}{2} \hat{m}_{f} \sqrt{\hat{m}_{f}^{2}+4 n^{2}}} .
$$

The mass scale is $\hat{m}_{f}=m_{0}\left(\frac{2 a_{1}}{a_{0}}\right)^{\frac{1}{2}} p^{+} \alpha^{\prime}$. For $n=0$ we have six modes with frequency $\hat{m}_{f}$ and then we get the two zero-modes we expected.

Let us now look at the MN case. For this background, the spectrum of fermionic oscillators is much easier to compute. These oscillators come in two sets of four with frequencies

$$
\begin{aligned}
& \omega_{n}^{++\beta}=\omega_{n}^{--\beta}=\sqrt{n^{2}+\frac{4}{9}\left(m_{0} p^{+} \alpha^{\prime}\right)^{2}}, \\
& \omega_{n}^{-+\beta}=\omega_{n}^{+-\beta}=\sqrt{n^{2}+\frac{1}{9}\left(m_{0} p^{+} \alpha^{\prime}\right)^{2}} .
\end{aligned}
$$

where $\beta=1$ or 2 and the signs $( \pm \pm)$ represent eigenvalues $\pm \frac{1}{2}$ under rotations in the $u$ and $v$ planes respectively. We have chosen to label the fermionic oscillations via these eigenvalues to illustrate a subtle yet simple point about the action of the original susy's from the MN solutions.

As we noted at the end of Sec. 3, the current $J=-\frac{1}{3} J_{1}+J_{2}+J_{\psi}$ used in the MN plane-wave limit is not in the global symmetry group $S U(2) \times S U(2)$ of the full theory. Correspondingly the Hamiltonian for the corresponding plane wave does not commute with the original four $N=1$ supercharges. On the other hand, the operator $J^{\prime}=J-\frac{2}{3} J_{1}=J-\frac{2}{3} J_{u}$ is an element of the original global symmetry group, so if we shift the Hamiltonian by $-\frac{2}{3} J_{u}$ we should get two zero frequency modes. Taking a careful look at (5.13) we see that this shift takes

$$
\omega_{n}^{+-\beta} \rightarrow \sqrt{n^{2}+\frac{1}{9}\left(m_{0} p^{+} \alpha^{\prime}\right)^{2}}-\frac{1}{3}\left(m_{0} p^{+} \alpha^{\prime}\right)
$$

giving the two required zero-modes for $n=0$. Thus, our string theory does exhibit the supersymmetry of the field theory, but it makes it somewhat hard to see.

Now that we described the spectrum of the fermionic Hamiltonian for MN and KS, we should make clear a few important connections with the bosonic Hamiltonian. First, if we define fermionic number operators

$$
N_{L}^{f}=\sum_{n=1}^{\infty} n\left(S_{n}^{\alpha \dagger} S_{n}^{\alpha}\right), \quad N_{R}^{f}=\sum_{n=1}^{\infty} n\left(\tilde{S}_{n}^{\alpha \dagger} \tilde{S}_{n}^{\alpha}\right),
$$

the contribution of the bosonic and fermionic modes is constrained by the equality of the overall occupation numbers [26] which have to satisfy the level-matching condition $N_{R}+\mathcal{N}_{R}+N_{R}^{f}=$ $N_{L}+\mathcal{N}_{L}+N_{L}^{f}$. Second, we note that in both cases the sum of the squares of the fermionic frequencies above exactly matches the sum of the squares of the frequencies of the bosonic fields in (5.1) order by order in $n$. This allows the corresponding string-theory to remain finite. Finally, neither the MN case nor the KS case has any linearly-realized worldsheet supersymmetries. 
This implies that there will be a zero-point energy for the overall Hamiltonian. Since at each level the sum of the fermionic frequencies is bigger than the sum of the bosonic frequencies, we will get a positive zero-point energy; there is no tachyon.

To conclude, the fermionic contribution to the Hamiltonian incorporates quite well our knowledge of the supersymmetries, especially for KS. The MN and KS pp-wave string theories are solvable, finite, and built on a positive-energy vacuum.

\section{$6 \quad$ A string theory of hadrons}

In order to interpret the Hamiltonian above in terms of the field theory dual to the "parent" background we must keep in mind the following facts. Local inertial momenta $P_{i}$, as measured in the string frame near $r=r_{0}$, are related to momenta in the field theory, $\mathcal{P}_{i}$, via the relation

$$
\mathcal{P}_{i}=g_{i i}\left(r_{0}\right) P_{i}
$$

We can also write the confined theory string tension, $T_{s}$, in terms of the string length as

$$
T_{s}=\left(g_{t t}\left(r_{0}\right) g_{x x}\left(r_{0}\right)\right)^{\frac{1}{2}} \frac{1}{\alpha^{\prime}}=g_{t t}\left(r_{0}\right) \frac{1}{\alpha^{\prime}} .
$$

Now eq. (5.7) can be written purely in terms of field theory variables as:

$$
H=\left[\frac{\mathcal{P}_{i}^{2}}{2 m_{0} J}+\frac{T_{s}}{2 m_{0} J}\left(\mathcal{N}_{R}+\mathcal{N}_{L}\right)\right]+\left[\frac{T_{s}}{2 m_{0} J}\left(H_{0}+H_{R}+H_{L}\right)\right]
$$

\subsection{The toy model of a string on a compact circle}

Before discussing the interpretation of these hadrons in the KS and MN theories, we begin by noting that there is a simple toy model ${ }^{4}$ — a string moving on a compact circle — which shares some parts of this Hamiltonian. As such, it helps to orient us toward a clear interpretation of the physics, although it does not capture all of the features of the Hamiltonian in (6.3). We simply consider a closed unwound string on flat $\mathcal{M}^{9} \times S^{1}$, the circle having radius $R_{0}$.

First consider an excited string at rest. Its energy is

$$
\sqrt{\frac{1}{\alpha^{\prime}}\left(N_{L}+N_{R}\right)}
$$

where (ignoring worldsheet fermions)

$$
N_{R}=\sum_{n=1}^{\infty} n\left(a_{n}^{i \dagger} a_{n}^{i}\right), \quad N_{L}=\sum_{n=1}^{\infty} n\left(\tilde{a}_{n}^{i \dagger} \tilde{a}_{n}^{i}\right)
$$

except that we sum over all 8 noncompact directions $x^{1}, \ldots, x^{8}$ transverse to a light cone (which we place in the directions $\left.x^{0}, x^{9}\right)$.

${ }^{4}$ M.J.S. thanks Minxin Huang, Thomas Levi, and Asad Naqvi for discussions concerning this toy model prior to the present work. 
Now let us boost the string, giving it small momentum $\overrightarrow{\mathbf{P}}$ in three spatial Minkowski directions and enormous momentum $P_{9} \equiv J / R$ in the $x^{9}$ direction. Its energy is now

$$
\sqrt{P_{9}^{2}+\overrightarrow{\mathbf{P}}^{2}+\frac{1}{\alpha^{\prime}}\left(N_{L}+N_{R}\right)}
$$

and so

$$
E-P_{9}=\frac{\overrightarrow{\mathbf{P}}^{2}}{2 J / R_{0}}+\frac{1}{2 J / R_{0}} \frac{1}{\alpha^{\prime}}\left(N_{L}+N_{R}\right)
$$

which looks similar to the formula (6.3) above if we identify $R_{0}$ as $1 / m_{0}$, and $T_{s}$ as $\frac{1}{\alpha^{\prime}}$.

How should we interpret this? From the ten-dimensional point of view, this is merely Lorentzian physics. But from the nine-dimensional point of view, we are adding not momentum but $K K$ charge to the string, whereby it remains static but becomes heavy. Any additional motion of the string in the noncompact directions looks like nonrelativistic motion from the point of view of nine dimensions. Excitations of the boosted string, which look perfectly ordinary from the ten-dimensional point of view, take the above squared form from the ninedimensional point of view. From this we learn that the first terms in (5.7) and (6.3) simply reflect how large and heavy nonrelativistic strings move and oscillate. ${ }^{5}$

Thus we are led to guess that the hadrons described in our string theory take the physical shape of nonrelativistic strings propagating in four dimensions. ${ }^{6}$ Since these hadrons have never been studied before, they need a name: we will call them "annulons" from the Latin word "anulus" for "ring." The vacuum of the string theory is the lowest-lying, stable annulon with charge $J$, and our string theory describes its motion and its small oscillations (as well as some other annulons to be discussed below.)

We will leave the toy model at this point, and return to gauge theory; but clearly this toy model will be a useful tool for obtaining additional physical insights into interactions, solitons, scaling laws, decay rates, etc. Some simple examples are given in our concluding section.

\subsection{The hadrons in the KS case}

The Hamiltonian (5.7) has a natural interpretation as describing a sector of the hadronic spectrum of the gauge theory. We will first discuss this in the context of the KS theory, which is easiest to interpret.

\footnotetext{
${ }^{5}$ Other related toy models can easily be found; for example, one might consider lifting the toy model to M-theory, and through an 11-9 flip relating the oscillations on a boosted string to strings on a bound state of $D_{0}$ branes. Indeed the Hamiltonian for excitations of such a bound state will look very similar to the first terms in our Hamiltonian. In all cases, it is the effect of tacking on a small oscillation to a large mass by addition in quadrature. Indeed Hamiltonians of this type have appeared many times in the contexts of DLCQ and strings with large winding number.

${ }^{6}$ More precisely, highly excited hadrons in our string theory actually "look" like strings. Low-lying states are small, essentially point particles, in the same way that gravitons in ordinary string theory do not look like strings but instead have well-localized wave functions. The wave functions for our low-lying hadrons can be guessed from those of ordinary strings, using the toy model.
} 


\subsubsection{The constituents in the KS theory}

To understand the hadrons in question, we need to understand the various objects that carry charge in the gauge theory. The massless fields of the gauge theory are those of pure $\mathcal{N}=1$ $S U(M)$ Yang-Mills; these are neutral under all anomaly-free $U(1)$ symmetries. However, as discussed in the appendix of [14], there are massive fields left over from the duality cascade. There are four chiral supermultiplets in the adjoint representation of $S U(M)$, charged as $(\mathbf{2}, \mathbf{2})$ under $S U(2)_{\ell} \times S U(2)_{r}$.

These emerge in the following way. The second to last stage of the cascade involves the gauge group $S U(2 M) \times S U(M)$, with fields $A_{1}, A_{2}$ in the $(\mathbf{2} \mathbf{M}, \overline{\mathbf{M}})$ representation, and fields $B_{1}, B_{2}$ in the conjugate representation; these fields are doublets under $S U(2)_{\ell}$ and $S U(2)_{r}$ respectively. The gauge group $S U(2 M)$ has $2 M$ flavors, so it confines [24]; in this process $S U(M)$ is mainly a spectator to the $S U(2 M)$ dynamics. Among the resulting bound states are the four fields

$$
\left(N_{i j}\right)_{\beta}^{\alpha}=\left(A_{i}\right)_{a}^{\alpha}\left(B_{j}\right)_{\beta}^{a}-\frac{1}{M} \delta_{\beta}^{\alpha} \operatorname{tr}\left(A_{i} B_{j}\right)
$$

which are in the adjoint representation of the spectator $S U(M)$ group, with indices $\alpha, \beta$; indices $a$ are in the confining $S U(2 M)$ group. The superpotential

$$
W \propto \operatorname{tr}\left(A_{i} B_{j} A_{k} B_{\ell}\right) \epsilon^{i k} \epsilon^{j \ell}
$$

generates, after confinement, a mass term

$$
W \propto \operatorname{tr}\left(N_{11} N_{22}-N_{21} N_{12}\right)
$$

for the $N_{i j}$; the physical mass of the $N_{i j}$, as we will see, is of order $m_{0}$. We can then make hadrons out of these heavy fields. (Note that there are also fields $\operatorname{tr}\left(A_{i} B_{j}\right)$ which are singlets of $S U(M)$; these ordinary mesons will play no role in the hadrons we are about to discuss.) Since the mass term marries $N_{11}$ and $N_{22}$, we cannot distinguish between $N_{11}$ and $N_{22}^{\dagger}$. (In the same way, and for the same reason, we cannot distinguish right-handed bottom quarks from left-handed ones by their gauge and global quantum numbers. ${ }^{7}$

The vacuum described by the KS solution has the property that only the $\mathbf{Z}_{2 M}$ chiral symmetry is broken, so the $S U(2)_{\ell} \times S U(2)_{r}$ is still realized. However, the geodesic that we choose is generated by the $U(1)=T_{\ell}^{3}+T_{r}^{3}$ in the diagonal $S U(2)$ subgroup, which we have called $U(1)_{D}$. The field $N_{11}$ carries charge 1 under the $U(1)_{D} ; N_{22}$ carries charge -1 , and $N_{12}$ carries charge 0 . Under the other symmetry left unbroken by the choice of geodesic, namely $U(1)_{A}=T_{\ell}^{3}-T_{r}^{3}$, $N_{11}$ and $N_{22}$ carry charge $0, N_{12}$ carries charge 1 and $N_{21}$ charge -1 .

\subsubsection{The lowest-lying annulon of charge $J$}

Consider the lowest-lying hadron of large charge $J$ built from $J$ of the constituents $N_{11}$, i.e. the state of lowest energy created by applying the operator $\operatorname{tr}\left(\left[N_{11}\right]^{J}\right)$ to the true $J=0$ vacuum

\footnotetext{
${ }^{7}$ Of course, the $N_{i j}$ are not the only bound states from the $S U(2 M)$ process, or indeed from the multiple steps of the duality cascade of KS. However, they are likely to be the only light stable multiplets, as would pions be in the absence of the electroweak interactions. The other bound states are also in the adjoint and in other representations neutral under the center of $S U(M)$; as such they have a marginal impact on the annulons.
} 
$|\Omega\rangle$ of the gauge theory

$$
\operatorname{tr}\left[\left(N_{11}\right)^{J}\right]|\Omega\rangle .
$$

This is the natural candidate for the vacuum $|0\rangle$ of our string theory Hamiltonian (5.7). As we have seen, $-P^{-}=H=E-m_{0} J=0$ in the vacuum, where $E$ is the eigenvalue of $i \partial_{t}$, the usual Minkowski Hamiltonian. Our vacuum state does therefore represent a state in the gauge theory with energy $M_{0} \equiv m_{0} J$, and as it has no other quantum numbers or degrees of freedom, it is natural to interpret it as the lowest-lying spin-zero hadron of charge $J$. As such it will contain a minimal number of constituents, namely $J$ of the heavy scalar $N_{11}$ particles and nothing else (except some ambient superglue, formed from the masless fields of the $\mathcal{N}=1$ $S U(M)$ SYM theory.)

We identify the mass of each $N_{11}$, in the mean field of all the others, as $m_{0}$. Because of collective effects among the particles, $m_{0}$ need not be the same as the mass appearing in the superpotential (which is holomorphic) or even the physical nonholomorphic mass given by canonically normalizing $N_{i j}$ in the effective Lagrangian for the gauge theory. Only from string theory do we learn that the average mass per $N_{11}$ is of the same order as glueball masses in the gauge theory, namely $m_{0}$. From the gauge theory this is a highly nonperturbative result. We know of no way to derive it, and indeed it may not be true at small 't Hooft coupling.

\subsubsection{The annulon in linear nonrelativistic motion}

Of course this hadron can move, and we should be able to write its kinetic energy. The first term in the Hamiltonian represents its nonrelativistic motion

$$
\frac{\mathcal{P}_{i}^{2}}{2 m_{0} J}=\frac{|\overrightarrow{\mathcal{P}}|^{2}}{2 M_{0}} .
$$

We should not be surprised that we obtain only the nonrelativistic kinetic energy; we will only be considering energies which are parametricaly smaller than $J$, so the kinetic energy will generally be much less than the mass $M_{0}$. We see the three worldsheet fields $x^{i}$ are required to be massless so that the spatial momenta of the hadron can be correctly represented. This feature is presumably generic; it shows the above string theory represents a compactification of string theory down to three non-compact spatial dimensions.

\subsubsection{Ripples on the annulon}

That this is really a four-dimensional string (or, more precisely, a five-dimensional string compactified and viewed under dimensional reduction to four dimensions) is indicated by the

$\mathcal{N}_{R}+\mathcal{N}_{L}$ term in the Hamiltonian. Since there are three noncompact spatial directions, the hadron will have stringy excitations in these directions which should be controlled by the oscillator modes on the worldsheet in the usual way. This is clearly the nature of this term.

Note that the spacing between the modes is not equal to the square root of the tension $T_{s}$ of the confining flux tube of the gauge theory, $\sqrt{T_{s}} \sim \sqrt{g M} m_{0}$, times the square root of the oscillator level $N$. Instead we find $g M m_{0} N / J$. From the form of the term in the Hamiltonian, it is natural to interpret this as tension $T_{s} \sim g M m_{0}^{2}$ divided by the mass of the hadron $M_{0}=m_{0} J$. 
This form is precisely what emerges in the above toy model and justifies our interpreting these hadrons as annulons, taking not only the mathematical form but also the physical shape of a heavy string.

\subsubsection{Insertion of constituents controlled by symmetries}

We can guess one more of the terms in the bosonic Hamiltonian on simple grounds. We know there is a hadron in the gauge theory which is the lowest lying state created by applying

$$
\operatorname{tr}\left(\left[N_{11}\right]^{J+1}\right)|\Omega\rangle
$$

where again $|\Omega\rangle$ is the vacuum of the gauge theory (not our ground-state annulon!) This differs from the lowest-lying annulon of charge $J$ only through the replacement $J \rightarrow J+1$, up to possible $1 / J$ corrections which are small at large $J$. In particular, we know this hadron has mass $m_{0}(J+1)$ for large $J$.

Now, an $S U(2)_{\ell}$ rotation of this state can convert it to a hadron of equal mass created by applying

$$
\operatorname{tr}\left(\left[N_{11}\right]^{J} N_{21}\right)|\Omega\rangle
$$

to the vacuum. This state, which carries $U(1)_{D}$ charge $J$ and $U(1)_{A}$ charge -1 , and differs in mass from our ground-state annulon by exactly $m_{0}$, should appear in our string theory.

Of course this is also true for $S U(2)_{r}$, which gives us an annulon with an inserted $N_{12}$. Can we insert an $N_{22}$ particle by acting first with $S U(2)_{\ell}$ and next with $S U(2)_{r}$ ? We can see the answer is no from two points of view. First, suppose we do act with the two $S U(2)$ symmetries in succession. Starting with an annulon with $J+2 N_{11}$ constituents, the action of $S U(2)_{\ell}$ gives us an annulon with one $N_{21}$ constituent, as in (6.14) above. The action of $S U(2)_{r}$ then gives us a hadron of the form

$$
\frac{1}{\sqrt{J}}\left[\sum_{k=1}^{J-1} \operatorname{tr}\left(\left[N_{11}\right]^{k} N_{12} N_{11}^{J-k} N_{21}\right)|\Omega\rangle+\operatorname{tr}\left(N_{11}^{J+1} N_{22}\right)|\Omega\rangle\right]
$$

Thus we obtain a state which predominantly has two new constituents, one each of $N_{12}$ and $N_{21}$. We see that the symmetries which relate the individual $N_{i j}$ to one another act rather differently on hadrons that already contain large numbers of $N_{11}$ particles.

Alternatively, suppose we add an $N_{22}$ particle into an annulon by hand; what happens to

$$
\operatorname{tr}\left(N_{11}^{J+1} N_{22}\right)|\Omega\rangle ?
$$

We claim there is no stable hadron which has a large overlap with this vector in the Hilbert space. The reason is dynamical. Although an individual $N_{22}$ particle is stable, in the presence of many $N_{11}$ particles it can easily convert via $N_{11} N_{22} \rightarrow N_{12} N_{21}$. The underlying process involves the term $\left|\partial W / \partial A_{1}\right|^{2}=\left|B_{1} A_{2} B_{2}\right|^{2}$ term in the Lagrangian, which allows $B_{1} A_{2} B_{2} \rightarrow B_{2} A_{2} B_{1}$ with $A_{1}$ as a spectator. (Alternatively this process can occur as $N_{22} N_{11} \rightarrow N_{12} N_{21}$, using the $\left|A_{2} B_{2} A_{1}\right|^{2}$ interaction.) Once this conversion takes place, the $N_{12}$ and $N_{21}$ can separate from one another within the annulon, and the chance of them recombining into an $N_{22}$ is phase-space suppressed - clearly of order $1 / J$. 
Similarly, if we act multiple times with $S U(2)_{\ell}$ and/or $S U(2)_{r}$ (adding $N_{11}$ particles so that their number, and the $U(1)_{D}$ charge, remain equal to $J$,) we obtain hadrons with arbitrary numbers of $N_{12}$ and $N_{21}$ particles inserted, but no $N_{22}$ particles, as long as the number of inserted constituents is small compared to $J$. From the symmetry arguments we know the masses of these hadrons differ from our vacuum annulon by integer units of $m_{0}$, that they carry integer charges under $U(1)_{A}$, and that they have no string oscillation modes excited (as they are related by symmetry to a vacuum annulon.) Therefore we predict the existence of two worldsheet operators with $U(1)_{A}$ charge \pm 1 which can insert an $N_{12}$ or $N_{21}$ particle into the annulon; these should be related by a symmetry, and should change the mass of the hadron by exactly $m_{0}$. This expectation is borne out, as discussed after Eq. (5.7). The $v, \bar{v}$ world-sheet fields, which descend from the part of the $S^{3}$ transverse to the geodesic, have precisely the right charges. The non-oscillatory mode associated to $v, \bar{v}$, applied on the string theory vacuum, leaves the $U(1)_{D}$ charge unchanged but gives $H=-P^{-}=m_{0}$, or $E=m_{0}(J+1)$, with $U(1)_{A}$ charge \pm 1 , as predicted.

We notice, from this structure, that the nondiagonal $S U(2)_{\ell}$ and $S U(2)_{r}$ generators are not operators in our string theory, because they carry nonzero $P^{+}$- they are charged under $U(1)_{D}$. Rather, they are operators which connect the Hilbert space of the string theory of charge $P^{+}=J$ to that with charge $P^{+}=J \pm 1$. In this way these symmetries can remain exact in the full theory but be absent within any one charge sector.

\subsubsection{Supersymmetry}

Since the gauge theory is $\mathcal{N}=1$ supersymmetric, we expect that the ground-state annulon (and indeed every state in the Hilbert space of the bosonic Hamiltonian) has a fermionic superpartner, a massive fermion, with the same mass and charge (and thus the same $P^{-}$.) Indeed, as the annulon is massive and charged, its multiplet structure is that of a complex multiplet, in particular the combination of a chiral multiplet of charge $J$ and a chiral multiplet of charge $-J$. This implies two complex scalar fields and a Dirac fermion (the same as the electronselectron supermultiplet in SQED.) In a dual string picture, the charge $J$ components of the complex multiplet can be generated using the zero modes of two massless worldsheet fermions on a state of fixed $p^{+}$. Taking into account the left-moving and right-moving contributions to the closed string Hilbert space, these zero-modes form two raising and two lowering operators generating a four-dimensional Hilbert space: two bosonic states and two fermionic. (Note our vacuum annulon is a complex boson.) As noted in section 5.2, two is precisely the number that we have. The action of one raising zero mode converts an $N_{11}$ constituent to its $\psi_{11}^{\alpha}$ fermionic partner ( $\alpha$ a spin index), and thus

$$
\operatorname{tr}\left[N_{11}^{J}\right]|\Omega\rangle \Rightarrow \operatorname{tr}\left[N_{11}^{J-1} \psi_{11}^{\alpha}\right]|\Omega\rangle
$$

Since we have the massless zero modes corresponding to the insertion of $\psi_{11}$, and since we have bosonic modes of mass $m_{0}$ corresponding to the insertion of $N_{12}$, why do we not have a fermionic mode of mass $m_{0}$ associated to $\psi_{12}$ ? The absence of this operator follows from the same logic (6.14)-(6.16) that explains the absence of a mode for $N_{22}$. We might expect that the combination of supersymmetry and $S U(2)_{r}$ would turn $N_{11} \rightarrow \psi_{12}$, but in a hadron 
with $J$ constituents the action of these two symmetries instead preferentially inserts one $N_{12}$ constituent and one $\psi_{11}$ constituent. Similarly, the transition $\psi_{12} N_{11} \rightarrow N_{12} \psi_{11}$ can be mediated by $S U(2 M)$-gluino exchange among the $A_{i}, B_{j}$ particles and their fermionic partners; as before, once this transition occurs the $\psi_{12}$ particle is unlikely to be reconstituted.

The same logic applies to the action of two supersymmetries; rather than generating the highest component of the $N_{11}$ chiral supermultiplet, the double action of supersymmetry inserts two $\psi_{11}$ constituents into the annulon.

\subsubsection{The non-universal directions}

The remaining structure of the Hamiltonian - the $z$ and $u, \bar{u}$ zero modes and the excited states in the five internal directions — cannot be predicted by any symmetry arguments. The only additional feature determined by symmetry is that in KS the transformation $S U(2)_{\ell} \leftrightarrow S U(2)_{r}$ which exchanges $v$ and $\bar{v}$ should be accompanied by $u \leftrightarrow-\bar{u}$ and $z \leftrightarrow-z$; but this symmetry is absent in MN. For this reason we expect the remaining features of the string theory to differ from model to model, and indeed the MN and KS cases are different.

We have made a number of attempts to interpret the non-universal directions in the KS theory. There are multiple possibilities, motivated by a variety of different arguments. However, we have been unable to determine which of these possible interpretations is correct, if any. We leave this issue for further research.

One important additional comment is that we have been a bit cavalier in specifying our hadrons. While it is true that our ground state hadron is the lowest-lying hadron created by $\operatorname{tr}\left(N_{11}^{J}\right)$ acting on the vacuum, it is not true that the hadron contains only $N_{11}$ particles. It may also contain $N_{22}^{\dagger}$ particles, and its wave function involves some mixture of possible states. (In a similar way, a proton may be created by $u_{L} u_{L} d_{L}$ or by $u_{R} u_{R} d_{R}$; the true proton has a wave function rather different from that suggested by either of these operators.) We have not determined the wave function of the ground state hadron unambiguously, and an understanding of the non-universal directions may require further investigation of this issue. Certainly there need not be any simple relation between the operators associated with the conformal conifoldderived pp-wave and the states associated with our pp-wave for the confining KS theory.

\subsubsection{Summary}

We now largely understand what our string theory is describing. The vacuum is a long, stable annulon of massive $N_{11}$ (and $N_{22}^{\dagger}$ ) particles. This heavy object can move in rectilinear nonrelativistic motion, and it can wriggle as a non-relativistic string. We can also insert into the chain of these particles any number of $N_{12}$ and $N_{21}$ particles. The original and inserted objects have a probability amplitude for their locations on the annulon, described by a wave function. The various energy eigenstates for this wave function give various hadronic states which correspond to strings with various excitations in the massive directions. The zero modes of the massive directions correspond to inserting the fields $N_{i j}$, and their conjugates, with constant amplitude around the string. This is of course consistent with BMN, but differs from 
it just as one would expect a description of states to differ from a description of operators. ${ }^{8}$

\subsection{More on the MN case}

Now let us turn to the MN case. We have already seen that the KS case has a geodesic direction, two $v$ directions, two $u$ directions, a $z$ direction and three $x^{i}$ directions. The $x^{i}$ directions are massless, from translational symmetry in real space, and the $v$ 's have mass $m_{0}$, from the $S U(2)$ symmetries which rotate the geodesic into the rest of the $S^{3}$. Also, there are two massless fermionic zero modes, by supersymmetry. The MN theory must share all of these features, which follow simply from symmetries, and as discussed in section 5, it does, though the supersymmetry is somewhat obscured.

The MN case involves the dimensional reduction of the six-dimensional $\mathcal{N}=2$ Yang-Mills theory on a two-sphere, with the appropriate twisting to maintain $\mathcal{N}=1$ supersymmetry in four dimensions. The massless six-dimensional $\mathcal{N}=2$ vector multiplet can be split into a six-dimensional $\mathcal{N}=1$ vector multiplet and a six-dimensional $\mathcal{N}=1$ hypermultiplet; the supercharge which survives the two-sphere reduction is in this $\mathcal{N}=1$ sub-algebra. After reduction, the massless six-dimensional $\mathcal{N}=1$ vector multiplet gives a massless four-dimensional $\mathcal{N}=1$ vector multiplet, along with a tower of massive Kaluza-Klein $\mathcal{N}=1$ vector multiplets in the adjoint representation. The lowest of these are three massive $\mathcal{N}=1$ vector multiplets in a triplet of $S U(2)_{r}$.

The other part of the six-dimensional $\mathcal{N}=2$ vector multiplet, the six-dimensional $\mathcal{N}=1$ hypermultiplet, gives only massive Kaluza-Klein modes after reduction on the two-sphere. These are all in four-dimensional complex $\mathcal{N}=1$ chiral multiplets and will transform under both $S U(2)$ symmetries. The lightest of these modes form two massive complex $\mathcal{N}=1$ chiral multiplets (eight real scalars) which transform in the bi-fundamental of $S U(2)_{\ell} \times S U(2)_{r}$. Thus both the KS and MN gauge theories have scalars corresponding to motion on the three-sphere at the minimum $A d S$ radius. In the MN case these motions are generated by the (now twisted) R-symmetry of the six-dimensional theory, while in KS they are the angular modes which rotate the $N_{11}$ particles into $N_{12}$ and $N_{21}$. The symmetries of the three-sphere are enough to predict that the properties of the $v, \bar{v}$ world-sheet fields in the MN case are the same as they are in the KS example.

For those phenomena not controlled by symmetries, the theories may, and do, differ. In particular, they differ on the masses $\hat{m}_{z}$ and $\hat{m}_{u}$, even when we account for the shift in mode frequencies discussed in section 5.2, which is needed to see the fermionic zero modes and supersymmetry. As discussed at the end of section 3, the MN case lacks the left-right symmetry of the KS case. Meanwhile the KS case has a surviving nonzero NS-NS 2-form, which the MN case lacks. All of these effects contribute to the differences between the two string theories.

The MN case does pose an additional problem: the $z$ direction is massless. Note, however, that this does not necessarily mean that it corresponds to a massless constituent, nor do we

\footnotetext{
${ }^{8}$ The structure of (5.7) also hints at a possible analogue of the non-relativistic quark model. In such a model, the stringy excitations in the massless directions could be interpreted as the insertion of "constituent" gluons into the ground-state annulon. For the massive directions, the excitations would simply involve insertions of $N_{12}, N_{21}$, etc. We leave this idea for future investigation.
} 
need to treat it as we treat the massless $x^{i}$ directions. Rather, it simply means that there is an excitation internal to an MN annulon which costs energy minus charge (both of which could be nonzero) much less than $m_{0}$ as $J \rightarrow \infty$. At the very least we expect this apparent flat direction, which clearly is not present in the full MN metric, to be lifted at finite $J$; this is in contrast to the $x^{i}$ directions which are massless by translational symmetry. This issue deserves further investigation however.

With the exception of this issue, the KS and MN theories are qualitatively similar in most respects and quantitatively equal where they ought to be. It would be interesting to compare these theories to the $\mathcal{N}=1^{*}$ case, if the latter is tractable.

\section{Wilson loops with charge}

In this section we return to the question of confinement and states of large $J$. We should be able to see that the theory is confining by examining Wilson loops. In the dual ten-dimensional supergravity, a straight tube of electric flux appears as a fundamental string extended in one of the Minkowski spatial directions and placed at a definite $A d S$ radius $r$ (and at a definite point in the other coordinates as well.) Such a string will fall to smaller $r$ unless dynamically prevented from doing so. In a conformal theory it can fall to $r=0$, where its tension, proportional to $\sqrt{g_{t t} g_{x x}}$, goes to zero [18]. In a confining gauge theory there is a minimum value of $\sqrt{g_{t t} g_{x x}}$, at some radius $r_{0}$. The string plummets to this radius but can go no further; its tension is bounded from below $[19,21]$. A heavy quark-antiquark pair at $\overrightarrow{\mathbf{x}}=(0,0,0)$ and $\overrightarrow{\mathbf{y}}=(\ell, 0,0)$ in the gauge theory, and the chromoelectric flux between them, correspond to a single string which ends on the boundary $r \rightarrow \infty$ of the gravity background at the points $\overrightarrow{\mathbf{x}}$ and $\overrightarrow{\mathbf{y}}$ [18]. The energy $V(\ell)$ of the configuration in the gauge theory is proportional to the total energy of the string (note it is formally infinite because the quark masses are infinite, but $d V / d \ell$ is finite.) If $d V / d \ell$ contains an $\ell$-independent additive constant $T$, then $V$ contains a term equal to $T \ell$, and the theory has linear confinement, with a flux tube of constant tension $T$. This happens precisely when the string with its ends fixed at $\overrightarrow{\mathbf{x}}$ and $\overrightarrow{\mathbf{y}}$ falls down to $r_{0}$ but can fall no further, and lies there like a rope resting in a flat-bottomed lake. Since the string lies entirely at $r_{0}$, we might expect to detect its tension by looking at geodesics near $r_{0}$.

But flux tubes of this sort know nothing about global charges, whereas we know that plane wave backgrounds involve considerations of highly-charged systems. How should we connect the two? One might think that one should consider quarks carrying global charge, but this is not correct. Instead, one should consider a quark-flux-antiquark system carrying large global charge. Such systems are not familiar from QCD, for a variety of reasons. However, in our theory, they are easy to construct, because we have massive adjoint fermions $\psi$ and scalars $\phi$ carrying a global $U(1)$ charge, with mass $m_{0}$. To such a gauge theory, we may add very heavy quarks $q\left(m_{q} \gg m_{0}\right)$ as probes of the system. We can then form heavy hadrons of the form $\bar{q} q, \bar{q} \phi q, \bar{q} \phi \phi q$, etc., of charge $0,1,2$, etc. Starting with a hadron $\bar{q}(\phi)^{J} q$, we can form a string labelled by $J$, its total charge — not its charge density! - by pulling the $q$ and $\bar{q}$ in the hadron until they are a distance $\ell$ apart. Our system is then made from a $q$, a $\bar{q}$, some gluons, $(n+J) \phi$ particles and $n \bar{\phi}$ particles, where $n$ may be fluctuating but $J$ is constant. The lowest energy 


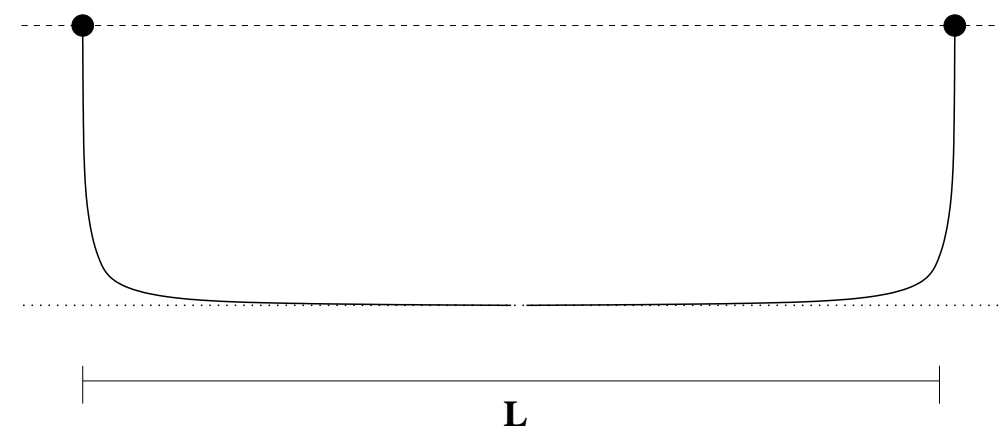

Figure 1: In the string dual of a confining theory, the quark and antiquark sources are given by the $r$-dependent segments near the ends of the string, while the flux tube between them is given by the region of the string lying at $r=r_{0}$.

eigenstate of this system has energy $V(\ell ; J)$, a function one might attempt to calculate. For $\ell$ very large and $J$ held finite, the effect of the $J$ widely-scattered $\phi$ particles will be negligible, and one will find $V(\ell, J) \approx 2 m_{q}+T \ell$. Conversely, for finite $\ell$ but with $J$ taken very large, one will find $V(\ell ; J)$ of order $2 m_{q}+J m_{0}$. Certainly, then, by looking at null geodesics at $r=r_{0}$, and looking at large $J$ but even larger $\ell$, we can detect whether there are confining flux tubes in the theory. But we will not have to work so hard. Instead, working at large $J$ and looking at the leading $\ell / J$ correction to $V(\ell, J)$, we will show the tension $T$ is nonzero.

In our theory, we can identify the $\phi$ particles with $N_{11}$. If $J$ is very large, so that the $N_{11}$ particles are very densely distributed on the flux tube, then we would expect these globallycharged flux tubes to be made from annulonic material. We would naturally guess, from our string theory, that just as excited states of the lowest-lying annulon have energy quadratic in the string tension, so $V(\ell ; J)$ will be quadratic in $T$ for very large $J$.

In fact, a semiclassical field theory analysis can already suggest for us the form that we should find. Let $T$ be the tension of a confining string with $J=0$. If the mass of the adjoint particles is $m_{0}$, we might guess the mass of a string of length $\ell$ would be $T \ell+J m_{0}$. But this is not correct; it does not account for the binding of the particles to the string. Instead, we must treat the the global charge that the string can carry in much the way one treats the electric charge of dyons - using collective coordinates.

We may get insight by taking a partly S-dual version of this problem, in which we bind heavy particles of mass $m_{0}$ with a conserved global charge to a magnetic flux tube. A still easier version is given by compactifying such a problem on a circle, so that the magnetic flux tubes themselves become particles (vortices in $2+1$ dimensions) and the binding problem becomes familiar. The vortices are solitons and the global charge they carry should be treated as in any collective coordinate problem, leading semiclassically to a formula for a soliton of vortex charge $p$ and global charge $q$ :

$$
M_{p, q}=\sqrt{p^{2} m_{\text {vortex }}^{2}+q^{2} m_{0}^{2}}
$$


just as with dyons in four dimensions. Lifting the problem back to four dimensions we replace $m_{\text {vortex }}$, with $T R$ (where $R$ is the radius of the compact dimension.) Indeed this can be seen in string theory, where one could consider various string-brane semiclassical bound state formulas, which are always governed by quadratic Born-Infeld formulas.

Following this logic, one is led to suspect that a similar formula governs the binding of heavy particles to an electric flux tube

$$
V(\ell ; J)=\sqrt{T^{2} \ell^{2}+J^{2} m_{0}^{2}} .
$$

This of course matches our naive expectations in the large $\ell$ and large $J$ limits. And for large $J$, we have

$$
V(\ell ; J) \approx J m_{0}+\frac{1}{2} \frac{T^{2} \ell^{2}}{J m_{0}},
$$

another "non-relativistic" formula. From this we learn that we can detect confinement in the gauge theory by looking at the order $-\ell^{2}$ term - not at an order- $\ell$ term! — in $V(\ell ; J)$. In general this formula might be subject to nonlinear corrections, but since we are in a limit where strings behave classically, we would not be surprised if such corrections were absent.

Of course this is also what is obtained in our toy model. Suppose we take a string on $M^{8} \times S^{1} \times S^{1}$, where the radii of the circles are $R_{1}$ and $R_{2}$, and we wrap the string on one circle while boosting it in the other. Before boosting, the string has mass $R_{1} / \alpha^{\prime}$. When boosted by $J$ units of KK momentum (not momentum per unit length) the string appears, from the nine-dimensional point of view, to be a static string of mass

$$
\sqrt{\left(\frac{R_{1}}{\alpha^{\prime}}\right)^{2}+\left(\frac{J}{R_{2}}\right)^{2}}
$$

which matches the formula above, if we identify $1 / R_{2}=m_{0}, 1 / \alpha^{\prime}=T$ and $R_{1}=\ell$. Again, at large $J$, we see the first correction to $J / R_{2}$ is quadratic in $R_{1}$.

Finally, we now calculate this in the supergravity dual to the gauge theory, by generalizing the results of [18]. In a confining theory, the energy of a system consisting of a heavy quark and a heavy antiquark at a distance $\ell$ from one another, and with no global charges, is given in supergravity by the total energy of a semiclassical string whose endpoints contact the boundary at spatial positions $\overrightarrow{\mathbf{x}}=(0,0,0)$ and $\overrightarrow{\mathbf{y}}=(\ell, 0,0)$. When $\ell$ is very large, and the theory is confining, the string becomes very simple, as shown in figure 1 . At the two ends, the string descends rapidly from the $A d S$ boundary to the radius $r_{0}$ where the tension of the string is minimized. This behavior is $\ell$-independent and the energy of these two regions correspond to the constant masses of the heavy quark and antiquark. The majority of the string lies along a line from $\overrightarrow{\mathbf{x}}=(0,0,0)$ to $\overrightarrow{\mathbf{y}}=(\ell, 0,0)$ but lying at $r=r_{0}$. The tension of the string in this region is constant, so the energy of this part of the string grows linearly with $\ell$; the constant of proportionality gives the tension of a confining string in the gauge theory.

Our claim is that the addition of global charge to this system is as simple as taking the above configuration and giving it a definite momentum along one of the $S^{1}$ directions in the compact five-dimensional space. We will now show that we reproduce the above expectations for $V(\ell ; J)$. 
We start by considering the Nambu-Goto action:

$$
S=\frac{1}{2 \pi \alpha^{\prime}} \int d \tau d \sigma \sqrt{-\operatorname{det} G_{M N} \partial_{\alpha} X^{M} \partial_{\beta} X^{N}}
$$

The general form of the classical configuration we are interested in involves an open string with its ends at two points on the boundary of the bulk space. Its radial position varies with $\sigma$, and it has in general nontrivial motion along an angle $\phi$. In short, the string has a worldsheet of the form

$$
t=t(\tau), \quad x=x(\sigma), \quad \phi=\phi(\tau), \quad r=r(\sigma) .
$$

The relevant part of the metric is therefore,

$$
d s^{2}=-g_{t t} d t^{2}+g_{x x} d x^{2}+g_{r r} d r^{2}+g_{\phi \phi} d \phi^{2}+\ldots
$$

Evaluating the Nambu-Goto action on this background we obtain

$$
S=\frac{1}{2 \pi \alpha^{\prime}} \int d \tau d \sigma \sqrt{\left(g_{t t} \dot{t}^{2}-g_{\phi \phi} \dot{\phi}^{2}\right)\left(g_{x x}+g_{r r}\left(\partial_{\sigma} r\right)^{2}\right)}
$$

The energy and angular momentum (global charge), as conjugate variables to $t$ and $\phi$, are

$$
\begin{aligned}
E & =\frac{1}{2 \pi \alpha^{\prime}} \int d \sigma g_{t t} \dot{t}\left(\frac{\left.g_{x x}\left(\partial_{\sigma} x\right)^{2}+g_{r r}\left(\partial_{\sigma} r\right)^{2}\right)}{g_{t t} \dot{t}^{2}-g_{\phi \phi} \dot{\phi}^{2}}\right)^{1 / 2}, \\
J & =\frac{1}{2 \pi \alpha^{\prime}} \int d \sigma g_{\phi \phi} \dot{\phi}\left(\frac{\left.g_{x x}\left(\partial_{\sigma} x\right)^{2}+g_{r r}\left(\partial_{\sigma} r\right)^{2}\right)}{g_{t t} \dot{t}^{2}-g_{\phi \phi} \dot{\phi}^{2}}\right)^{1 / 2} .
\end{aligned}
$$

The precise minimization of the energy subject to fixed charge $J$ would be quite involved in the backgrounds we consider. For example, for the KS background the warp factor is not known analytically for all values of the radius. However, using a natural ansatz, we can obtain an excellent estimate for the relationship between the energy and the angular momentum, and later justify how any contribution left unaccounted for is appropriately suppressed.

We naturally fix the static gauge $t=\tau$ and $x=\ell \frac{\sigma}{2 \pi}$, where $\ell$ is the total length of the string (as measured in the gauge theory, using the Minkowski metric!). Most of the string lies along the "minimal" radius $r_{0}$

$$
r[x(\sigma)]=r_{0}, \delta<x<\ell-\delta
$$

and $r[x] \rightarrow \infty$ as $x \rightarrow 0$ and as $x \rightarrow \ell$. We assume that $\delta$ is independent of $\ell$ for large $\ell$, which corresponds to the physical expectation that the quark and antiquark sources do not grow as $\ell$ increases. We also choose that the endpoints do not rotate, so that the quark and antiquark sources do not themselves carry any global charge. This means that $\dot{\phi} \rightarrow 0$ at the ends of the string at $\sigma=0,2 \pi$. Finally — and this is the least obvious part of the ansatz — we assume that the majority of the angular momentum is spread uniformly in the region far from the ends, by taking the majority of the string to move uniformly on a circle parameterized by $\phi$ :

$$
\phi \approx \omega \tau, \delta<x<\ell-\delta
$$


Altogether this implies

$$
J \approx \frac{\omega}{2 \pi \alpha^{\prime}} \sqrt{\left.\frac{g_{\phi \phi}^{2} g_{x x}(\ell / 2 \pi)^{2}}{g_{t t}-g_{\phi \phi} \omega^{2}}\right|_{r=r_{0}}} \int_{2 \pi \delta / \ell}^{2 \pi(1-\delta / \ell)} d \sigma \approx \frac{\omega \ell}{2 \pi \alpha^{\prime}} \sqrt{\left.\frac{g_{\phi \phi}^{2} g_{x x}}{g_{t t}-g_{\phi \phi} \omega^{2}}\right|_{r=r_{0}}} .
$$

With this gauge and ansatz we have

$$
\begin{aligned}
E \approx & \frac{1}{2 \pi \alpha^{\prime}} \int_{0}^{2 \pi \delta / \ell} d \sigma g_{t t}\left(\frac{\left.g_{x x}(\ell / 2 \pi)^{2}+g_{r r}\left(\partial_{\sigma} r\right)^{2}\right)}{g_{t t}-g_{\phi \phi} \dot{\phi}^{2}}\right)^{1 / 2} \\
& +\frac{1}{2 \pi \alpha^{\prime}} \int_{2 \pi(1-\delta / \ell)}^{2 \pi} d \sigma g_{t t}\left(\frac{\left.g_{x x}(\ell / 2 \pi)^{2}+g_{r r}\left(\partial_{\sigma} r\right)^{2}\right)}{g_{t t}-g_{\phi \phi} \dot{\phi}^{2}}\right)^{1 / 2} \\
& +\frac{1}{2 \pi \alpha^{\prime}}\left(\left.\frac{g_{t t} g_{x x}(\ell / 2 \pi)^{2}}{g_{t t}-g_{\phi \phi} \omega^{2}}\right|_{r=r_{0}}\right)^{1 / 2} \int_{2 \pi \delta / \ell}^{2 \pi(1-\delta / \ell)} d \sigma \\
\approx & 2 \mathbf{m}_{q}+\left.\frac{g_{t t}}{g_{\phi \phi}}\right|_{r=r_{0}} \frac{J}{\omega}
\end{aligned}
$$

where

$$
\mathbf{m}_{q} \equiv \frac{1}{2 \pi \alpha^{\prime}} \int_{0}^{2 \pi \delta / \ell} d \sigma g_{t t}\left(\frac{\left.g_{x x}(\ell / 2 \pi)^{2}+g_{r r}\left(\partial_{\sigma} r\right)^{2}\right)}{g_{t t}-g_{\phi \phi} \dot{\phi}^{2}}\right)^{1 / 2} \approx \frac{1}{2 \pi \alpha^{\prime}} \int_{0}^{2 \pi \delta / \ell} d \sigma \sqrt{g_{t t} g_{r r}}\left(\partial_{\sigma} r\right) .
$$

This last expression is divergent, representing the infinite mass of the heavy quark, but more importantly for our purposes it is essentially $\ell$-independent, and gives a physically inconsequential additive shift to the energy $V(\ell ; J)$.

More succinctly, defining

$$
m_{0} \equiv \lim _{r \rightarrow r_{0}} \sqrt{g_{t t} / g_{\phi \phi}}
$$

as we did both for KS and MN, we have

$$
J \approx \frac{g_{t t} \ell}{2 \pi \alpha^{\prime}} \frac{\omega / m_{0}^{2}}{\sqrt{1-\omega^{2} / m_{0}^{2}}}, E \approx 2 \mathbf{m}_{q}+\frac{g_{t t} \ell}{2 \pi \alpha^{\prime}} \frac{1}{\sqrt{1-\omega^{2} / m_{0}^{2}}} .
$$

whence

$$
V(\ell ; J)=E \approx 2 \mathbf{m}_{q}+\sqrt{\frac{g_{t t}^{2} \ell^{2}}{\left(2 \pi \alpha^{\prime}\right)^{2}}+m_{0}^{2} J^{2}}
$$

Recalling that the tension is $T=\sqrt{\left|g_{t t} g_{x x}\right|} /\left(2 \pi \alpha^{\prime}\right)=g_{t t} / 2 \pi \alpha^{\prime}$, we reproduce the formula (7.2) obtained by the field theory analysis:

$$
V(\ell ; J) \approx \sqrt{T^{2} \ell^{2}+m_{0}^{2} J^{2}}+\text { constant. }
$$

In an appendix, we show that our ansatz is stable and that all corrections are of order $1 / \ell$ or $1 / J$ relative to the terms that we have kept. 


\section{Closing Comments}

We have found a sector of a gauge theory whose hadrons are described by an exactly soluble string theory. We obtained them through a Penrose limit around a geodesic sitting at the minimum $A d S$ radius, moving in Minkowski time, and winding around a circle on the compact part of the bulk space. We obtained a description of hadrons of charge $J$ with mass of order $J$, which we argued were of the form of nonrelativistic strings. The string theory describes their motion, their ripples, their superpartners and their global symmetry partners.

It is important to emphasize a mathematically essential point that makes our construction possible. One of the key characteristics of the Einstein equations is their nonlinearity, which implies that the expansion in the metric around a neighborhood of a particular point is not a well-defined procedure. For example, in the context of the KS background, the Ricci flatness of the six-dimensional space perpendicular to the Minkowski directions is required in order to satisfy the equations of motion. The deformed conifold is, of course, Ricci flat. However, truncating the metric near the apex of the deformed conifold to second order results in a space metrically equivalent to $\mathbf{R}^{3} \times S^{3}$ which is no longer Ricci flat and thus ceases to satisfy the equations of motion. The use of such approximations is equivalent to neglecting back-reaction in many situations, and although one might extract sensible results it is not a consistent procedure in general.

In the context of the Penrose limit [8-10] however, there is a well-defined expansion around a null geodesic. Properly interpreted, this amounts to having to consider the expansion up to second order around the null geodesic. In particular, in the KS solution, expanding the metric around the end of the conifold at $\tau=0$ (here $r \propto \cosh \tau$ ), keeping up to quadratic terms in $\tau$, is a well-defined truncation in the Penrose limit. This particular property of the limit makes our analysis exact.

It is interesting, and at first glance slightly puzzling, that we have recovered particles moving in three spatial dimensions by taking a Penrose limit. It was argued in [30] that the boundary of the pp-wave corresponding to conformal field theories is a null line - null in the bulk but timelike from the coordinates of the field theory. In short, the theory is purely quantum mechanical, with no spatial dimensions. Why, then, do we seem to have spatial directions in our limit? In fact, we do not have them. Although we have three spatial momenta, which can take any finite values, we are working in a limit where the annulon masses are infinite, and thus the spatial velocities are all zero. Thus the annulons never actually move anywhere (at infinite $J$ ), and the theory remains quantum mechanical despite the presence of continuous momenta.

This feature, and many others, is captured by our toy model of a string boosted along a compact circle. This model can further be used to guess other dynamical features of these particles. For example, the stability of the annulons to decay can easily be estimated. An annulon of charge $J$ can decay to two annulons of charge $J_{1}$ and $J_{2}$. The rate for this process is the same as that for a one-to-two string decay in flat space, but greatly slowed down in the lab frame by the time dilation associated with the boost. Similarly, the rate for two-totwo scattering is simply given by the Virasoro-Shapiro amplitude and its generalizations. For example, if annulons of charge $J_{1}$ and $J_{2}$ scatter elastically, the amplitude is given by a sum over $s$-channel annulon poles of charge $J_{1}+J_{2}$, or equivalently by a sum over ordinary strings 
of charge $J=0$ in the $t$-channel. Regge physics and other characteristic features of string amplitudes will also be reproduced.

Another initially puzzling observation is that the original BMN string theory describes operators with $J^{2} \ll N$, and has an effective coupling constant $g N / J^{2}$. But where BMN finds this latter combination, we find $g M / J$, or, more accurately, $(g M)^{2} / J^{2}$. This phenomenon is a reflection of an important property of the KS metric: the number of colors in the far infrared, as measured by the integral of the 3 -form through the $S^{3}$, is $M$, but the far-infrared metric has each factor of $M$ enhanced by an extra factor of $(g M)$. Essentially, the dual to $\mathcal{N}=4 \mathrm{SYM}$ (or to the far UV of $\mathrm{KS}$ ) is controlled mainly by a relation between the metric and the 5-form, but in the far infrared this converts to a relation between the metric and the 3 -form. In the process the extra factor of $g M$ appears.

Thus, in our string theory, the effective coupling constant is $g[g M] M / J^{2}$, and the subleading effects which are being neglected are of order $J^{2} /[g M] M$. This latter point is very important, because it shows that our analysis is only good when $J \ll \sqrt{g} M \ll M$. Consequently our strings, despite having $J$ scaling like $M$, are still far from being baryon-like giant gravitons (with $J \sim M$.)

A related question involves the properties of annulons at small 't Hooft coupling. In this regime some aspects of the annulons are presumably described using field theoretic perturbation theory, and those of the charged Wilson loops via a semiperturbative treatment. At present we do not know which aspects of our results continue to this regime, and how the remainder are modified. There are many interesting issues to be considered here, not the least of which is identifying the difference between annulons and giant-gravitonic hadrons - the latter being well-described using Hartree-Fock mean-field techniques, as in Witten's description of large- $N$ baryons.

Finally, we would hope that this set of hadrons, which do not appear in QCD, are not the only ones which can be treated in this fashion. States of high spin and small charge, which are long semiclassical strings far along Regge trajectories, do appear in Yang-Mills theory, and to some degree in physical QCD. It is these states which appear in the original Chew-Frautschi plots of the so-called Regge trajectories of QCD. Any improvement in our ability to study these states would be of substantial physical interest.

\section{Acknowledgments}

We have benefited from discussions with D. Berenstein, S. Cherkis, J. Figueroa-O'Farrill, J. Gomis, A. Hashimoto, C. Herzog, I. Klebanov, J. Maldacena, A. Naqvi, C. Nùñez, N. Seiberg, C. Thorn and A. Tseytlin. J.S. and M.J.S. thank the Institute for Advanced Study, where much of this work was done. E.G.G., L.A.P-.Z and M.J.S are grateful to the Aspen Center for Physics for hospitality at various stages of this work. E.G.G. is supported by Frank and Peggy Taplin, and by NSF grant PHY-0070928. L.A.P.-Z. is supported by a grant in aid from the Funds for Natural Sciences at I.A.S. The work of J.S was supported in part by the US-Israel Binational Science Foundation and by the Israel Science Foundation. M.J.S was supported by DOE grant DOE-FG02-95ER40893, NSF grant PHY-0070928, and an award by the Alfred P. Sloan Foundation. 


\section{A Deformed Conifold: Metric and Symmetries}

\section{A.1 New Coordinates for the Deformed Conifold}

To find a convenient set of coordinates, we go back to the basics [22]. The deformed conifold is defined in terms of a complex two-by-two matrices $W$ satisfying:

$$
\operatorname{det} W=-\frac{\epsilon^{2}}{2}
$$

We define the variable $\tau$ by setting:

$$
\operatorname{tr}\left(W^{\dagger} W\right)=\epsilon^{2} \cosh \tau
$$

A simple solution to this equation is

$$
W_{\epsilon}=\frac{\epsilon}{\sqrt{2}}\left(\begin{array}{cc}
e^{\frac{\tau}{2}} & 0 \\
0 & -e^{\frac{-\tau}{2}}
\end{array}\right)=\frac{\epsilon}{\sqrt{2}} e^{\frac{\tau}{2} \sigma_{3}} \sigma_{3},
$$

and we can generate the whole set by acting on the left and right with $S U(2)$ matrices $L$ and $R$ :

$$
W=L W_{\epsilon} R^{\dagger}
$$

For reasons which will soon be obvious, we choose to re-write the two $S U(2)$ matrices $L$ and $R$ as:

$$
L=T S, \quad R=\sigma_{3} S \sigma_{3} .
$$

Now for $\tau \neq 0$ we see that if we take

$$
S \rightarrow S e^{i \theta \sigma_{3}}
$$

then $W$ remains invariant. This means that $S$ represents coordinates for $S U(2) / U(1)=S^{2}$. Also, when $\tau=0, W$ takes the form

$$
W=T S W_{0} \sigma_{3} S^{\dagger} \sigma_{3}=T W_{0}=T \sigma_{3}
$$

which means that $T \in S U(2)=S^{3}$ is a good coordinate for the $S^{3}$ at the origin and gives a coordinate independent of $S$ for $\tau \neq 0$.

We will now compute the deformed conifold metric in these new coordinates, starting from $[22,23]$ :

$$
d s_{6}^{2}=\epsilon^{-\frac{2}{3}} K(\tau) \operatorname{tr}\left(d W^{\dagger} d W\right)+\epsilon^{-\frac{8}{3}} \sinh ^{-1}(\tau) K^{\prime}(\tau)\left|\operatorname{tr}\left(W^{\dagger} d W\right)\right|^{2} .
$$

We first write

$$
T^{\dagger} d T=-d T^{\dagger} T=\frac{i}{2} \omega^{a} \sigma_{a}, \quad S=e^{\frac{i}{2} \phi \sigma_{3}} e^{-\frac{i}{2} \theta \sigma_{2}}
$$


and then

$$
\begin{aligned}
\operatorname{tr}\left(d W^{\dagger} d W\right)= & \frac{\epsilon^{2}}{4} \cosh (\tau)\left(d \tau^{2}+\left(\omega^{a}\right)^{2}\right) \\
+ & \epsilon^{2} \sinh ^{2}\left(\frac{\tau}{2}\right)\left[\left(d \theta^{2}+\sin ^{2} \theta d \phi^{2}\right)\right. \\
& -\left(\sin \phi \omega^{1}+\cos \phi \omega^{2}\right)(d \theta) \\
& \left.-\left(\cos \theta \cos \phi \omega^{1}-\cos \theta \sin \phi \omega^{2}-\sin \theta \omega^{3}\right)(\sin \theta d \phi)\right]
\end{aligned}
$$

and

$$
\begin{aligned}
\left|\operatorname{tr}\left(W^{\dagger} d W\right)\right|^{2}=\frac{\epsilon^{4}}{4} \sinh ^{2}(\tau)\left[d \tau^{2}+\right. & \\
& \left.\left(\sin \theta \cos \phi \omega^{1}+\sin \theta \sin \phi \omega^{2}+\cos \theta \omega^{3}\right)^{2}\right]
\end{aligned}
$$

\section{A.2 Connection to Other Coordinates}

We would like to connect our coordinates above with the ones used in [14] (inherited from Minasian and Tsimpis [23]). These coordinates can be written in the following manner:

$$
\begin{aligned}
& W=L_{1} W_{\epsilon} \sigma_{3} \sigma_{1} L_{2}^{\dagger}, \\
& L_{1}=e^{\frac{i \phi_{1}}{2} \sigma_{3}} e^{\frac{-i \theta_{1}}{2} \sigma_{2}} e^{\frac{i \psi}{4} \sigma_{3}}, \\
& L_{2}=e^{\frac{i \phi_{2}}{2} \sigma_{3}} e^{\frac{-i \theta_{2}}{2} \sigma_{2}} e^{\frac{i \psi}{4} \sigma_{3}} .
\end{aligned}
$$

We can now rewrite the 1 -forms $g^{i}$ derived from these coordinates in terms of the 1 -forms which come from $T^{\dagger} d T$ and $S^{\dagger} d S$. One first writes a change of basis for the 1 -forms $\omega^{i}$,

$$
\sqrt{2} \tilde{g}^{3} \sigma_{1}-\sqrt{2} \tilde{g}^{4} \sigma_{2}+\tilde{g}^{5} \sigma_{3}=S^{\dagger} \omega^{a} \sigma_{a} S
$$

which gives

$$
\begin{aligned}
\tilde{g}^{5} & =\sin \theta \cos \phi \omega^{1}-\sin \theta \sin \phi \omega^{2}+\cos \theta \omega^{3} \\
-\tilde{g}^{4} & =\frac{1}{\sqrt{2}}\left(\sin \phi \omega^{1}+\cos \phi \omega^{2}\right) \\
\tilde{g}^{3} & =\frac{1}{\sqrt{2}}\left(\cos \theta \cos \phi \omega^{1}-\cos \theta \sin \phi \omega^{2}-\sin \theta \omega^{3}\right) .
\end{aligned}
$$

After a little algebraic work one finds

$$
\begin{aligned}
e^{-\frac{i}{2} \psi}\left(g^{3}+i g^{4}\right) & =\tilde{g}^{3}+i \tilde{g}^{4} \\
g^{5} & =\tilde{g}^{5} \\
e^{-\frac{i}{2} \psi}\left(g^{1}+i g^{2}\right) & =\left(\tilde{g}^{3}-\sqrt{2} \sin \theta d \phi\right)+i\left(\tilde{g}^{4}+\sqrt{2} d \theta\right)
\end{aligned}
$$


Now we can rewrite the expression (A.10) and (A.11) as

$$
\begin{aligned}
\operatorname{tr}\left(d W^{\dagger} d W\right) & =\frac{\epsilon^{2}}{4} \cosh (\tau)\left[d \tau^{2}+\left(\tilde{g}^{5}\right)^{2}+2\left(\left(\tilde{g}^{3}\right)^{2}+\left(\tilde{g}^{4}\right)^{2}\right)\right] \\
& +\frac{\epsilon^{2}}{2} \sinh ^{2}\left(\frac{\tau}{2}\right)\left[\left(\left(\tilde{g}^{1}\right)^{2}+\left(\tilde{g}^{2}\right)^{2}\right)-\left(\left(\tilde{g}^{3}\right)^{2}+\left(\tilde{g}^{4}\right)^{2}\right)\right] \\
\left|\operatorname{tr}\left(W^{\dagger} d W\right)\right|^{2} & =\frac{\epsilon^{4}}{4} \sinh ^{2}(\tau)\left[d \tau^{2}+\left(\tilde{g}^{5}\right)^{2}\right]
\end{aligned}
$$

If we now use the fact that

$$
\frac{1}{3 K^{2}(\tau)}=\frac{1}{2} \cosh (\tau) K(\tau)+\frac{1}{2} \sinh (\tau) K^{\prime}(\tau)
$$

and plug back into (A.8), we recover the metric (4.2)

$d s_{6}^{2}=\frac{1}{2} \varepsilon^{4 / 3} K(\tau)\left[\frac{1}{3 K^{3}(\tau)}\left(d \tau^{2}+\left(g^{5}\right)^{2}\right)+\cosh ^{2}\left(\frac{\tau}{2}\right)\left[\left(g^{3}\right)^{2}+\left(g^{4}\right)^{2}\right]+\sinh ^{2}\left(\frac{\tau}{2}\right)\left[\left(g^{1}\right)^{2}+\left(g^{2}\right)^{2}\right]\right]$.

\section{B String theory Hamiltonian}

The equations of motion following from the worldsheet Lagrangian of section 5 are

$$
\begin{aligned}
& \eta^{\alpha \beta} \partial_{\alpha} \partial_{\beta} x^{i}=0, \\
& \eta^{\alpha \beta} \partial_{\alpha} \partial_{\beta} z-\left(m_{0} \phi^{+} \alpha^{\prime}\right)^{2}\left(\frac{4 a_{1}}{a_{0}}-\frac{4}{5}\right) z=0, \\
& \eta^{\alpha \beta} \partial_{\alpha} \partial_{\beta} u_{1}-\left(m_{0} \phi^{+} \alpha^{\prime}\right)^{2}\left(\frac{4 a_{1}}{a_{0}}-\frac{3}{5}\right) u_{1}-\sqrt{2} m_{0} p^{+} \alpha^{\prime}\left(\frac{a_{1}}{a_{0}}\right)^{1 / 2} \partial_{\sigma} v_{2}=0, \\
& \eta^{\alpha \beta} \partial_{\alpha} \partial_{\beta} v_{2}-\left(m_{0} \phi^{+} \alpha^{\prime}\right)^{2} v_{2}+\sqrt{2} m_{0} p^{+} \alpha^{\prime}\left(\frac{a_{1}}{a_{0}}\right)^{1 / 2} \partial_{\sigma} u_{1}=0, \\
& \eta^{\alpha \beta} \partial_{\alpha} \partial_{\beta} u_{2}-\left(m_{0} \phi^{+} \alpha^{\prime}\right)^{2}\left(\frac{4 a_{1}}{a_{0}}-\frac{3}{5}\right) u_{2}-\sqrt{2} m_{0} p^{+} \alpha^{\prime}\left(\frac{a_{1}}{a_{0}}\right)^{1 / 2} \partial_{\sigma} v_{1}=0, \\
& \eta^{\alpha \beta} \partial_{\alpha} \partial_{\beta} v_{1}-\left(m_{0} \phi^{+} \alpha^{\prime}\right)^{2} v_{1}+\sqrt{2} m_{0} p^{+} \alpha^{\prime}\left(\frac{a_{1}}{a_{0}}\right)^{1 / 2} \partial_{\sigma} u_{2}=0,
\end{aligned}
$$

The Fourier expansion for generic closed-string classical solutions $z$ can be written as

$$
\begin{aligned}
z(\sigma, \tau) & =i \sqrt{\frac{\alpha^{\prime}}{2}}\left[\frac{1}{\sqrt{\hat{m}_{z}}}\left(a_{0}^{z} e^{-2 i \hat{m}_{z} \tau}-a_{0}^{i \dagger} e^{2 i \hat{m}_{z} \tau}\right)\right. \\
& \left.+\sum_{n=1}^{\infty} \frac{1}{\sqrt{w_{n}^{z}}}\left[e^{-2 i w_{n}^{z} \tau}\left(a_{n}^{i} e^{2 i n \sigma}+\tilde{a}_{n}^{i} e^{-2 i n \sigma}\right)-e^{2 i w_{n}^{z} \tau}\left(a_{n}^{i \dagger} e^{-2 i n \sigma}+\tilde{a}_{n}^{i \dagger} e^{2 i n \sigma}\right)\right]\right]
\end{aligned}
$$

where $w_{n}^{z}=\sqrt{n^{2}+\hat{m}_{z}^{2}}$. We have conveniently normalized the $a$ 's such that their Poisson bracket is not proportional to the frequency. For the massless coordinates we have the standard 
expansion. To determined the frequencies of the coupled system we introduce the standard mode expansion

$$
u_{1}=\sum_{n} A_{n}^{1} e^{i\left(\omega_{n} \tau+n \sigma\right)}, \quad v_{1}=\sum_{n} B_{n}^{1} e^{i\left(\omega_{n} \tau+n \sigma\right)},
$$

and a similar ansatz for $u_{2}$ and $v_{2}$. Substituting the ansatze in the equations of motions give

$$
\left(\omega_{n}^{ \pm}\right)^{2}=\frac{1}{2}\left[2 n^{2}+\hat{m}_{v}^{2}+\hat{m}_{u}^{2} \pm \sqrt{\left(\hat{m}_{v}^{2}-\hat{m}_{u}^{2}\right)^{2}+4 n^{2} \hat{m}_{B}^{2}}\right]
$$

where

$$
\hat{m}_{v}=m_{0} p^{+} \alpha^{\prime}, \quad \hat{m}_{u}^{2}=\left(m_{0} p^{+} \alpha^{\prime}\right)^{2}\left(\frac{4 a_{1}}{a_{0}}-\frac{3}{5}\right), \quad \hat{m}_{B}=\sqrt{2} m_{0} p^{+} \alpha^{\prime}\left(\frac{a_{1}}{a_{0}}\right)^{1 / 2} .
$$

Note that the frequency of the zero modes are $\omega_{0}^{+}=\hat{m}_{v}$ and $\omega_{0}^{-}=\hat{m}_{u}$ and they correspond at the zero-mode level to excitations associated with $v$ 's and $u$ 's respectively. We therefore have three massless oscillators, one massive with mass $\hat{m}_{z}$, two massive with $\hat{m}_{v}$ and the final two with $\hat{m}_{u}$. As expected the effect of the B-field $\left(\hat{m}_{B}\right)$ appears only for the nonzero modes $(n \neq 0)$.

For the fermionic sector (for more detail, see for example $[12,26]$ ) we have two coupled Majorana-Weyl spinors $\theta^{1}$ and $\theta^{2}$ which satisfy a the lightcone gauge condition, $\Gamma_{+} \theta^{1,2}=0$, and obey the following equation of motion:

$$
\begin{aligned}
\left(\partial_{\tau}+\partial_{\sigma}\right) \theta^{1} & =-\frac{1}{8}\left(p^{+} \alpha^{\prime}\right) \Gamma^{i j}\left(F_{3}\right)_{+i j} \theta^{2}-\frac{1}{8}\left(p^{+} \alpha^{\prime}\right) \Gamma^{i j}\left(H_{3}\right)_{+i j} \theta^{1}, \\
\left(\partial_{\tau}-\partial_{\sigma}\right) \theta^{2} & =-\frac{1}{8}\left(p^{+} \alpha^{\prime}\right) \Gamma^{i j}\left(F_{3}\right)_{+i j} \theta^{1}+\frac{1}{8}\left(p^{+} \alpha^{\prime}\right) \Gamma^{i j}\left(H_{3}\right)_{+i j} \theta^{2} .
\end{aligned}
$$

In order to solve these equations, we combine the two spinors into one complex spinor $\epsilon$, fourier transform $\epsilon$ in the variable $\sigma$ and combine the two first order equations into one second-order equation. For the MN plane-wave we get

$$
\ddot{\epsilon}_{n}=-\left[n^{2}+\frac{m_{0}^{2}}{18}\left(5-3 \Gamma_{u_{1} u_{2} v_{1} v_{2}}\right)\right] \epsilon .
$$

which gives four fermionic oscillators each with $\Gamma_{u_{1} u_{2} v_{1} v_{2}}= \pm 1$ and frequencies

$$
\omega_{n}^{+}=\sqrt{n^{2}+\frac{\hat{m}_{0}^{2}}{9}}, \quad \text { and } \quad \omega_{n}^{-}=\sqrt{n^{2}+\frac{4 \hat{m}_{0}^{2}}{9}}
$$

with $\hat{m}_{0}=p^{+} \alpha^{\prime} m_{0}$. For the KS plane--wave we get the a slightly more complicated equation:

$$
\ddot{\epsilon}_{n}=-\left[n^{2}+\hat{m}_{f}^{2}+\left\{-\frac{1}{4} \hat{m}_{f}^{2}-n \frac{\hat{m}_{f}}{2}\left(i \Gamma_{u_{1} v_{2}}\right)-\frac{\hat{m}_{f}^{2}}{2}\left(i \Gamma_{u_{2} v_{2}}\right)\right\}\left(1+\Gamma_{u_{1} u_{2} v_{1} v_{2}}\right)\right] \epsilon
$$

with $\hat{m}_{f}=\left(2 a_{1} / a_{0}\right)^{\frac{1}{2}} m_{0} p^{+} \alpha^{\prime}$. If we expand our spinors in a \pm 1 eigenbasis of $\left(i \Gamma_{u_{1} u_{2}}\right)$ and $\left(i \Gamma_{u_{1} u_{2}}\right)$ we can write down four pairs of fermionic oscillators. The frequencies for the first two pairs have the simple form

$$
\omega_{n}^{++}=\omega_{n}^{--}=\sqrt{n^{2}+\hat{m}_{f}^{2}} .
$$


while the spinors with eigenvalues $(+-)$ and $(-+)$ mix. To get their eigen-frequencies we diagonalize the matrix

$$
\left[\begin{array}{cc}
\left(n^{2}+\frac{1}{2} \hat{m}_{f}^{2}\right) & -\left(i n \hat{m}_{f}+\frac{1}{2} \hat{m}_{f}^{2}\right) \\
\left(i n \hat{m}_{f}-\frac{1}{2} \hat{m}_{f}^{2}\right) & \left(n^{2}+\frac{1}{2} \hat{m}_{f}^{2}\right)
\end{array}\right]
$$

which yields the frequencies

$$
\omega_{n}^{\prime \prime}=\sqrt{n^{2}+\frac{1}{2} \hat{m}_{f}^{2} \pm \frac{1}{2} \hat{m}_{f} \sqrt{\hat{m}_{f}^{2}+4 n^{2}}} .
$$

Note that $\omega_{0}$ "- is a zero-frequency mode .

\section{Stability of the Wilson loop ansatz}

Let us now turn to the question of the reliability of the results on the properties of the Wilson loop as a function of $L$ and $J$. The first important issue we clarify is the stability of the classical configuration. This is most easily done in the Polyakov formulation of the string. We thus consider the nonlinear sigma model

$$
S=\frac{1}{4 \pi \alpha^{\prime}} \int d^{2} \sigma G_{i j} \partial_{a} X^{i} \partial^{a} X^{j}
$$

In the conformal gauge, the equation of motion for $X^{a}$ being $t, \phi$ or $x$ has the same form:

$$
\partial_{\alpha}\left(g_{a a} \eta^{\alpha \beta} \partial_{\beta} X^{a}\right)=0 .
$$

They are all trivially satisfied. To understand the stability of the solution we consider the linear fluctuations from the equations of motion. Since $\partial_{r} g_{a a} \mid-r_{0}=0$ we see that there is no mixing between the radial fluctuation and the fluctuations along $\phi$ and $x$. Next we note that the resulting equation for the fluctuations $\left(\phi=\phi_{0}+e^{i \nu \tau} \delta \phi(\sigma)\right)$ is simply

$$
\left(\partial_{\sigma}^{2}+\nu^{2}\right) \delta \phi(\sigma)=0
$$

We should now assume some boundary conditions for $\delta \phi(\sigma)$. The natural ones would be Dirichlet at the ends of the interval where our solution is reliable, i.e. in $\sigma=[\delta / \ell, 2 \pi-\delta / \ell]$. This implies that the spatial structure of the fluctuation is

$$
\delta \phi(\sigma)=\delta \phi_{n} \sin \left[\frac{n}{1-\frac{\delta}{\pi \ell}}\left(\sigma-\frac{\delta}{\ell}\right)\right] .
$$

Returning to (C.3) we see that all the frequencies are positive and therefore the solution is stable. The last important point is understanding the contribution of the edges, that is, the regions in which it is not appropriate to consider $\partial_{\sigma} r \approx 0$ since precisely in these regions the strings go between the boundary and the "minimal" radius. The effects of these edges have been considered in a similar setting in, for example, [31]. The estimate of [31] applied to our situation gives $\frac{d}{L} \sim \mathcal{O}\left(L^{-1}\right)$, where $d$ is the region along which $\partial_{\sigma} r$ cannot be approximated as zero. This means that in the large $L$ limit the contribution of these regions is correspondingly suppressed. Similarly we expect suppression of the order of $1 / J$. 


\section{References}

[1] J. Polchinski and M. J. Strassler, "Hard scattering and gauge/string duality," Phys. Rev. Lett. 88 (2002) 031601 [arXiv:hep-th/0109174].

[2] D. Berenstein, J. M. Maldacena and H. Nastase, "Strings in flat space and pp waves from N = 4 super Yang Mills," JHEP 0204 (2002) 013 [arXiv:hep-th/0202021].

[3] S. S. Gubser, I. R. Klebanov and A. M. Polyakov, "A semi-classical limit of the gauge/string correspondence," Nucl. Phys. B 636 (2002) 99 [arXiv:hep-th/0204051].

[4] R. R. Metsaev, "Type IIB Green-Schwarz superstring in plane wave Ramond-Ramond background," Nucl. Phys. B 625 (2002) 70 [arXiv:hep-th/0112044].

R. R. Metsaev and A. A. Tseytlin, "Exactly solvable model of superstring in plane wave Ramond-Ramond background," Phys. Rev. D 65 (2002) 126004 [arXiv:hep-th/0202109].

[5] N. Itzhaki, I. R. Klebanov and S. Mukhi, "PP wave limit and enhanced supersymmetry in gauge theories," JHEP 0203 (2002) 048 [arXiv:hep-th/0202153].

[6] J. Gomis and H. Ooguri, "Penrose limit of $\mathrm{N}=1$ gauge theories," Nucl. Phys. B 635 (2002) 106 [arXiv:hep-th/0202157].

[7] L. A. Pando Zayas and J. Sonnenschein, "On Penrose limits and gauge theories," JHEP 0205 (2002) 010 [arXiv:hep-th/0202186].

[8] R. Penrose, "Any space-time has a plane wave as a limit", in Differential Geometry and Relativity, Reidel, Dordrecht, 1976.

R. Penrose, "Techniques of Differential Topology in Relativity", SIAM,1972.

[9] R. Güven, "Plane wave limits and T-duality," Phys. Lett. B 482 (2000) 255 [arXiv:hepth/0005061].

[10] M. Blau, J. Figueroa-O'Farrill and G. Papadopoulos, "Penrose limits, supergravity and brane dynamics," arXiv:hep-th/0202111.

M. Blau, J. Figueroa-O'Farrill, C. Hull and G. Papadopoulos, "A new maximally supersymmetric background of IIB superstring theory," JHEP 0201 (2002) 047 [arXiv:hepth/0110242].

[11] E. G. Gimon, L. A. Pando Zayas and J. Sonnenschein, "Penrose limits and RG flows," arXiv:hep-th/0206033.

[12] D. Brecher, C. V. Johnson, K. J. Lovis and R. C. Myers, "Penrose limits, deformed ppwaves and the string duals of $\mathrm{N}=1$ large N gauge theory," arXiv:hep-th/0206045.

[13] J. M. Maldacena and C. Nunez, "Towards the large N limit of pure N = 1 super Yang Mills," Phys. Rev. Lett. 86 (2001) 588 [arXiv:hep-th/0008001]. 
[14] I. R. Klebanov and M. J. Strassler, "Supergravity and a confining gauge theory: Duality cascades and chiSB-resolution of naked singularities," JHEP 0008 (2000) 052 [arXiv:hepth/0007191].

[15] C. P. Herzog, I. R. Klebanov and P. Ouyang [C01-07-20.4 Collaboration], "Remarks on the warped deformed conifold," arXiv:hep-th/0108101.

C. P. Herzog, I. R. Klebanov and P. Ouyang, "D-branes on the conifold and $\mathrm{N}=1$ gauge / gravity dualities," arXiv:hep-th/0205100.

[16] J. Sonnenschein, "Stringy confining Wilson loops," arXiv:hep-th/0009146.

Y. Kinar, E. Schreiber and J. Sonnenschein, "Q anti-Q potential from strings in curved spacetime: Classical results," Nucl. Phys. B 566 (2000) 103 [arXiv:hep-th/9811192].

[17] V. E. Hubeny, M. Rangamani and E. Verlinde, "Penrose limits and non-local theories,' JHEP 0210 (2002) 020 [arXiv:hep-th/0205258]. Y. Oz and T. Sakai, "Penrose limit and sixdimensional gauge theories," Phys. Lett. B 544 (2002) 321 [arXiv:hep-th/0207223]. H. Fuji, K. Ito and Y. Sekino, "Penrose limit and string theories on various brane backgrounds," JHEP 0211 (2002) 005 [arXiv:hep-th/0209004]. S. Bhattacharya and S. Roy, "Penrose limit and NCYM theories in diverse dimensions," arXiv:hep-th/0209054. S. Bhattacharya and S. Roy, "More on Penrose limits and non-local theories," arXiv:hep-th/0210072. M. Alishahiha and A. Kumar, "PP-waves from nonlocal theories," JHEP 0209 (2002) 031 [arXiv:hep-th/0207257].

[18] J. M. Maldacena, "Wilson loops in large N field theories," Phys. Rev. Lett. 80 (1998) 4859 [arXiv:hep-th/9803002].

S. J. Rey and J. Yee, "Macroscopic strings as heavy quarks in large N gauge theory and anti-de Sitter supergravity," Eur. Phys. J. C 22 (2001) 379 [arXiv:hep-th/9803001].

[19] E. Witten, "Anti-de Sitter space, thermal phase transition, and confinement in gauge theories," Adv. Theor. Math. Phys. 2, 505 (1998) [arXiv:hep-th/9803131]; A. Brandhuber, N. Itzhaki, J. Sonnenschein and S. Yankielowicz, "Wilson loops, confinement, and phase transitions in large N gauge theories from supergravity," JHEP 9806, 001 (1998) [arXiv:hep-th/9803263]; D. J. Gross and H. Ooguri, "Aspects of large N gauge theory dynamics as seen by string theory," Phys. Rev. D 58, 106002 (1998) [arXiv:hep-th/9805129].

[20] A. H. Chamseddine and M. S. Volkov, "Non-Abelian BPS monopoles in N = 4 gauged supergravity," Phys. Rev. Lett. 79 (1997) 3343 [arXiv:hep-th/9707176].

[21] A. Loewy and J. Sonnenschein, "On the holographic duals of $N=1$ gauge dynamics," JHEP 0108, 007 (2001) [arXiv:hep-th/0103163].

[22] P. Candelas and X. C. de la Ossa, "Comments On Conifolds," Nucl. Phys. B 342 (1990) 246.

[23] R. Minasian and D. Tsimpis, "On the geometry of non-trivially embedded branes," Nucl. Phys. B 572 (2000) 499 [arXiv:hep-th/9911042]. 
[24] N. Seiberg, "Exact results on the space of vacua of four-dimensional SUSY gauge theories," Phys. Rev. D 49, 6857 (1994) [arXiv:hep-th/9402044].

[25] I. Bena and R. Roiban, "Supergravity pp-wave solutions with 28 and 24 supercharges," arXiv:hep-th/0206195.

[26] J. G. Russo and A. A. Tseytlin, "On solvable models of type IIB superstring in NS-NS and R-R plane wave backgrounds," JHEP 0204 (2002) 021 [arXiv:hep-th/0202179].

[27] M. Cvetic, H. Lu and C. N. Pope, "M-theory pp-waves, Penrose limits and supernumerary supersymmetries," Nucl. Phys. B 644 (2002) 65 [arXiv:hep-th/0203229].

[28] M. Cvetic, H. Lu, C. N. Pope and K. S. Stelle, "Linearly-realised worldsheet supersymmetry in pp-wave background," arXiv:hep-th/0209193.

[29] R. Corrado, N. Halmagyi, K. D. Kennaway and N. P. Warner, "Penrose limits of RG fixed points and pp-waves with background fluxes," arXiv:hep-th/0205314.

[30] D. Berenstein and H. Nastase, arXiv:hep-th/0205048.

[31] Y. Kinar, E. Schreiber, J. Sonnenschein and N. Weiss, "Quantum fluctuations of Wilson loops from string models," Nucl. Phys. B 583 (2000) 76 [arXiv:hep-th/9911123]. 